\title{
Pacific
}

Journal of

Mathematics

\section{SUPERTROPICAL LINEAR ALGEBRA}

ZUR IZHAKIAN, MANFRED KNEBUSCH AND LOUIS ROWEN 


\title{
SUPERTROPICAL LINEAR ALGEBRA
}

\author{
ZUR IZHAKIAN, MANFRED KNEBUSCH AND LOUIS ROWEN
}

\begin{abstract}
The objective of this paper is to lay out the algebraic theory of supertropical vector spaces and linear algebra, utilizing the key antisymmetric relation of "ghost surpasses". Special attention is paid to the various notions of "base", which include d-base and s-base, and these are compared to other treatments in the tropical theory. Whereas the number of elements in various d-bases may differ, it is shown that when an s-base exists, it is unique up to permutation and multiplication by scalars, and can be identified with a set of "critical" elements. Then we turn to orthogonality of vectors, which leads to supertropical bilinear forms and a supertropical version of the Gram matrix, including its connection to linear dependence. We also obtain a supertropical version of a theorem of Artin, which says that if g-orthogonality is a symmetric relation, then the underlying bilinear form is (supertropically) symmetric.
\end{abstract}

\section{Introduction}

The objective of this paper is to lay the foundations for an algebraic theory of linear algebra in supertropical mathematics. Special attention is paid to the notion of base, which plays a subtler role here than in classical linear algebra. Although an extensive literature already exists on tropical linear algebra over the max-plus algebra, including matrix rank [Akian et al. 2006] and linear dependence [Akian et al. 2009], the emphasis often is combinatoric or geometric. The traditional approach in semiring theory is to divide the determinant into a positive and negative part (since -1 need not exist in the semiring); see [Akian et al. 1990]. The ensuing reliance

The research of Izhakian and Rowen is supported by the Israel Science Foundation (grant no. 448/09). Research on this paper was carried out by the three authors through the Research in Pairs program of the Mathematisches Forschungsinstitut Oberwolfach (MFO), Germany, July 2009. The research of Izhakian was supported by the Oberwolfach Leibniz Fellows Programme, MFO. Knebusch was supported in part by the Gelbart Institute at Bar-Ilan University, the Minerva Foundation at Tel-Aviv University, the Mathematics Department of Bar-Ilan University, and the Emmy Noether Institute. The authors are grateful to Tal Perri for helpful comments on an earlier version.

MSC2010: primary 11D09, 15A03, 15A04, 15A15, 15A63, 16Y60; secondary 51M20, 20M18, 15A33, $14 \mathrm{~T} 05$.

Keywords: tropical algebra, supertropical vector spaces, linear algebra, change of base semirings,

linear and bilinear forms, Gram matrix. 
on combinatorics leads to competing (and different) definitions. For example, in [Akian et al. 2006], five different definitions of matrix rank are given: the row rank, the Barvinok (Shein) rank, the strong rank, the Gondran-Minoux rank, the symmetrized rank, and the Kapranov rank. Since [Maclagan and Sturmfels 2009] is an excellent source of fundamental results and examples, we use it as a general reference for the "standard" tropical theory and compare several of our notions with the definitions given there.

Extending the max-plus algebra to the algebra of [Izhakian and Rowen 2010] yields a theory paralleling the classical structure theory of commutative algebras. The structure theory of supertropical predomains tends to unify these notions, giving a single formula for the determinant, from which we can define a nonsingular matrix; then the row rank, column rank, and strong rank all coincide. This makes it easier to proceed with a traditional algebraic development. Explicit properties of matrices, especially singularity, were studied in [Izhakian and Rowen 2009; 2011a; 2011b; 2011c]. We elaborate a bit, by proving in Theorem 3.5 that the product of two nonsingular $n \times n$ matrices cannot be in $M_{n}\left(\mathscr{G}_{\mathbb{O}}\right)$ (although it might be singular).

Our main objective in this paper is to study bases of supertropical vector spaces over supertropical semifields, relying as far as possible on the structure theory. While this theory parallels the classical theory of linear algebra, several key differences do emerge. The major difference is that whereas in classical linear algebra, any maximal independent set is a minimal spanning set, these two notions differ significantly in the tropical theory. Thus, we must consider two kinds of "base", one defined by means of independence and the other defined by means of spanning.

First, one can take a maximal (tropically) independent set, which we call a $d$ base, called a "basis" in [Maclagan and Sturmfels 2009, Definition 5.2.4]. This has considerable geometric significance, intuitively providing a notion of rank (although, by an example in [Maclagan and Sturmfels 2009], the rank might vary according to the choice of d-base). As one might expect from [Izhakian and Rowen 2011b], any dependence among vectors can be extended to an (often unique) saturated dependence, which is maximal in a certain sense; see Theorem 4.20. This leads to a delicate analysis of the rank of a subspace, especially since it turns out that the number of elements in different d-bases may differ.

Alternatively, one may consider sets that (tropically) span the subspace; a minimal such set, when it exists, is called an s-base. Such sets are used in generating convex spaces, as studied in [Feichtner and Sturmfels 2005]. Not every d-base is an s-base, and not every s-base is a d-base. In fact, the number of elements of an s-base might necessarily be larger than the number of elements of a d-base. Surprisingly, an s-base is unique up to scalar multiples, by Corollary 5.25, and actually can be characterized in terms of critical elements, which intuitively are elements that cannot be decomposed into sums of other elements; see Theorem 5.24. On the 
other hand, the d-bases can be quite varied, and lead us to interesting subspaces that they span.

In the last section, we introduce supertropical bilinear forms in order to study "left ghost orthogonality" between vectors. One calls two vectors $v$ and $w$ left $g$-orthogonal with respect to a supertropical bilinear form $\langle$,$\rangle when \langle v, w\rangle$ is a ghost. We construct the Gram matrix and prove the connection between tropical dependence of vectors in a nondegenerate space and the singularity of their Gram matrix (Theorem 6.7). Finally, we prove Theorem 6.19, a variant of Artin's theorem: When the g-orthogonality relation is symmetric in the sense of Definition 6.12, the supertropical bilinear form is "supertropically symmetric".

\section{Supertropical structures}

Supertropical semifields. A semiring without zero, which we notate as a semiring ${ }^{\dagger}$, is a structure $\left(R,+, \cdot, \mathbb{1}_{R}\right)$ such that $\left(R, \cdot, \mathbb{1}_{R}\right)$ is a monoid and $(R,+)$ is a commutative semigroup, with distributivity of multiplication over addition on both sides. (In other words, a semiring ${ }^{\dagger}$ does not necessarily have the zero element $\mathbb{O}$, but any semiring can also be considered as a semiring ${ }^{\dagger}$.) Given a semiring ${ }^{\dagger} R^{\dagger}$, we can formally adjoin the element $\mathbb{O}$ to obtain the semiring $R:=R^{\dagger} \cup\{\mathbb{O}\}$, where we stipulate, for all $a \in R$,

$$
\mathbb{O}+a=a+\mathbb{O}=a, \quad \mathbb{Q} a=a \mathbb{0}=\mathbb{O} .
$$

A supertropical semiring ${ }^{\dagger}$ is a triple $\left(R^{\dagger}, \mathscr{G}, v\right)$, where $R^{\dagger}$ is a semiring ${ }^{\dagger}$ and $\mathscr{G}$ is a semiring ${ }^{\dagger}$ ideal called the ghost ideal, together with an idempotent map

$$
v: R^{\dagger} \rightarrow \mathscr{G}
$$

(preserving multiplication as well as addition) called the ghost map on $R^{\dagger}$, satisfying the properties

(a) $a+b=a^{v}$ if $a^{v}=b^{v}$, and

(b) $a+b \in\{a, b\}$ for all $a, b \in R^{\dagger}$ such that $a^{v} \neq b^{v}$. (Equivalently, $\mathscr{G}$ is ordered via $a^{\nu} \leq b^{v}$ if and only if $a^{\nu}+b^{\nu}=b^{\nu}$.)

In particular, $v(a)=a+a$. We write $a^{v}$ for $v(a)$.

Remark 2.1. The element $e:=v\left(\mathbb{1}_{R}\right)$ is both a multiplicative and additive idempotent of $R^{\dagger}$, which plays a key role, since $v\left(R^{\dagger}\right)=e R^{\dagger}$. Each element of $e R^{\dagger}$ is additively idempotent:

$$
e r+e r=(e+e) r=e r .
$$

We write $a>_{\nu} b$ if $a^{v}>b^{v}$.

Definition 2.2. Elements $a$ and $b$ of $\left(R^{\dagger}, \varphi, v\right)$ are $v$-matched, written $a \cong_{\nu} b$, if $a^{v}=b^{\nu}$. The element $a$ dominates $b$ if $a>_{\nu} b$. 
Recall that any commutative supertropical semiring satisfies the Frobenius formula from [Izhakian and Rowen 2010, Remark 1.1]:

$$
(a+b)^{m}=a^{m}+b^{m}
$$

for any $m \in \mathbb{N}^{+}$.

A supertropical semifield $d^{\dagger}$ is a supertropical semiring ${ }^{\dagger}\left(F^{\dagger}, \mathscr{G}, v\right)$ in which $\mathscr{T}:=$ $F^{\dagger} \backslash \mathscr{G}$ is an Abelian group and the restriction from $v$ to $\mathscr{T}$ maps onto $\mathscr{G} . \mathscr{T}$ is called the set of tangible elements of $F^{\dagger}$.

We have the analogous definitions when we adjoin the element $\mathbb{O}_{F}$ to the supertropical semifield ${ }^{\dagger} F^{\dagger}$ to obtain the supertropical semifield $F$. Thus, we write

$$
F:=F^{\dagger} \cup\left\{\mathbb{O}_{F}\right\}=\left(F, \mathscr{G}_{\mathbb{O}}, \nu\right)
$$

where $\mathscr{G}_{\mathbb{O}}:=\mathscr{G} \cup\left\{\mathbb{D}_{F}\right\}$ is the ghost ideal and the ghost map $v: F \rightarrow \mathscr{G}_{\mathbb{O}}$ satisfies $v\left(\mathbb{O}_{F}\right)=\mathbb{O}_{F}$. Conversely, given a supertropical semifield $\left(F, \mathscr{G}_{\mathbb{D}}, v\right)$, we can take $F^{\dagger}=F \backslash\left\{\mathbb{O}_{F}\right\}$ and $\mathscr{G}=\mathscr{G}_{\mathbb{0}} \backslash\left\{\mathbb{O}_{F}\right\}$ and define the supertropical semifield ${ }^{\dagger}\left(F^{\dagger}, \mathscr{G}, v\right)$. Thus, the theories with or without $\mathbb{O}_{F}$ are basically the same. Throughout the remainder of this paper, $F=\left(F, \mathscr{G}_{\mathbb{0}}, v\right)$ denotes a supertropical semifield.

Intuitively, the tangible elements correspond in some sense to the original maxplus algebra, although here $a+a=a^{v}$ instead of $a+a=a$. Our motivating example [Izhakian 2009] of a supertropical semifield, used as the primary example throughout [Izhakian and Rowen 2010] as well as in this paper, is the extended tropical semiring

$$
\mathbb{T}:=D(\mathbb{R}):=\left(\mathbb{R} \cup \mathbb{R}^{v} \cup\{-\infty\}, \mathbb{R}^{v} \cup\{-\infty\}, 1_{\mathbb{R}}\right),
$$

whose operations are induced by the standard operations max and + over the real numbers; we call this logarithmic notation, since the zero element $\mathbb{O}_{\mathbb{T}}$ is $-\infty$ and the unit element $\mathbb{1}_{\mathbb{T}}$ is 0 . To clarify our exposition, most of the examples in this paper are presented for $D(\mathbb{R})$. On the other hand, it is often convenient to take $\mathbb{Q}$ or $\mathbb{Z}$ instead of $\mathbb{R}$, especially when working with powers of elements, and the "characteristic 1" semifield" consisting of the single element $\mathbb{1}$ has interesting applications. (This becomes the well-known "Boolean semifield" $\{\mathbb{O}, \mathbb{1}\}$ when the zero element is adjoined.) Semirings of polynomials over supertropical semifields also play a crucial role in tropical geometry. Nonidempotent semifields ${ }^{\dagger}$ such as $\mathbb{Q}^{+}$have applications to arithmetic. Accordingly, our theory is framed for vector spaces over supertropical semifields ${ }^{\dagger}$, although, in analogy to the classical theory, the major theorems could also be formulated over semidomains.

The supertropical semifield plays a basic role in supertropical algebra parallel to the role of the field in classical algebra. Accordingly, one is led to study linear algebra over supertropical semifields. 
Occasionally, we also want to pass back from $\mathscr{G}$ to $\mathscr{T}$. Abusing notation slightly, we pick a representative in $\mathscr{T}$ for each class in the image of $\widehat{v}$, thereby getting a function $\widehat{v}: R^{\dagger} \rightarrow \mathscr{T}$ by putting $\left.\widehat{v}\right|_{\mathscr{T}}=1_{\mathscr{T}}$.

The "ghost surpass" and "ghost dependence" relations. We consider the supertropical semiring $\left(R, \mathscr{G}_{\mathbb{0}}, v\right)$.

Definition 2.3. We say $b$ is ghost dependent on $a$, written $b \curlyvee_{\mathrm{gd}} a$, if $a+b \in \mathscr{G}_{\mathbb{D}}$.

In particular, $a \cong_{\nu} b$ implies that $a \curlyvee_{\mathrm{gd}} b$.

The ghost dependence relation is symmetric, but not transitive, since $1 \curlyvee_{\mathrm{gd}} 3^{v}$ and $3^{v} \curlyvee_{\mathrm{gd}} 2$, although 1 and 2 are not ghost dependent. The following antisymmetric and transitive relation is a key to much of the theory.

Definition 2.4. We define the ghost surpasses relation $\models_{\mathrm{gs}}$ on a supertropical semiring $R=\left(R, \varphi_{\mathbb{0}}, v\right)$ by

$$
a \models_{\mathrm{gs}} b \Longleftrightarrow a=b+c \text { for some } c \in \mathscr{G}_{\mathbb{0}} \text {. }
$$

In this notation, by writing $a \models_{\mathrm{gs}} \mathbb{O}_{R}$ we mean $a \in \mathscr{G}_{\mathbb{0}}$. This restricts to the ghost surpasses relation on $R^{\dagger}$ by

$$
a \models_{\mathrm{gs}} b \Longleftrightarrow a=b \text { or } a=b+c \text { for some } c \in \mathscr{G} .
$$

Remark 2.5. The following are equivalent:

(1) $a \curlyvee_{\mathrm{gd}} \mathbb{O}_{R}$.

(2) $a \in \mathscr{G}_{0}$.

(3) $a \models_{\mathrm{gs}} \mathbb{O}_{R}$.

We quote some easy properties of $\models_{\mathrm{gs}}$ from [Izhakian and Rowen 2011b]:

Remark 2.6. (i) When $a$ is tangible, $a \models_{\mathrm{gs}} b$ implies $a=b$ [loc. cit., Remark 1.2]. In particular, tangible elements are comparable under $\models_{\mathrm{gs}}$ if and only if they are equal. In this way, the relation $\models_{\text {gs }}$ generalizes equality.

(ii) $a \models_{\mathrm{gs}} b$ if and only if $a=b$ or $a$ is a ghost $\geq_{\nu} b$. In particular, if $a \models_{\mathrm{gs}} b$ then $a \geq_{\nu} b$; if $a \models_{\mathrm{gs}} b$ for $b \in \mathscr{G}_{\mathbb{D}}$, then $a \in \mathscr{G}_{\mathbb{0}}$.

(iii) The relation $\models_{\mathrm{gs}}$ is a partial order on $R$ [loc. cit., Lemma 1.5].

(iv) If $a \models_{\mathrm{gs}} b$, then $a \Upsilon_{\mathrm{gd}} b$.

Supertropical vector spaces. Modules over semirings (often called "semimodules" in the literature, or sometimes "cones") are defined just as modules over rings, except that now the additive structure is that of a semigroup instead of a group. (Subtraction does not enter into the other axioms of a module over a ring.) 
Definition 2.7. Let $R$ be a semiring. An $R$-module $V$ is a semigroup $\left(V,+, \mathbb{O}_{V}\right)$ together with scalar multiplication $R \times V \rightarrow V$ satisfying the following properties for all $r_{i} \in R$ and $v, w \in V$ :

(1) $r(v+w)=r v+r w$.

(2) $\left(r_{1}+r_{2}\right) v=r_{1} v+r_{2} v$.

(3) $\left(r_{1} r_{2}\right) v=r_{1}\left(r_{2} v\right)$.

(4) $\mathbb{1}_{R} v=v$.

(5) $r \mathbb{O}_{V}=\mathbb{O}_{V}$.

(6) $\mathbb{O}_{R} v=\mathbb{O}_{V}$.

Note 2.8. One could also define a module over a semiring ${ }^{\dagger}$ by deleting Axiom (6). In the other direction, any module $V$ over a semiring ${ }^{\dagger} R^{\dagger}$ becomes an $R$-module when we formally define $\mathbb{O}_{R} v=\mathbb{O}_{V}$ for each $v \in V$.

The reason we prefer the terminology "module" is that this definition of module over a semiring $R$ coincides with the usual definition of module when $R$ is a ring, since $-v=\left(-\mathbb{1}_{R}\right) v$.

In case the underlying semiring $R$ is a supertropical semifield $F, V$ is called a (supertropical) vector space over $F$, or vector space for short. We focus on vector spaces in this paper, and call their elements vectors. Our main example of a vector space in this paper, as well as the main example in [Izhakian and Rowen 2011a], is $F^{(n)}$, whose ghost map acts as $v$ on each component. The zero element $\mathbb{Q}$ of $F^{(n)}$ is $\left(\mathbb{O}_{F}, \ldots, \mathbb{O}_{F}\right)$. However, we also are interested in subspaces of $F^{(n)}$, so we need this greater generality.

A supertropical vector space $V$ has the distinguished standard ghost submodule $\mathcal{H}_{\mathbb{D}}:=e V$, as well as the ghost map $v: V \rightarrow \mathscr{H}_{\mathbb{D}}$, given by $v(v):=v+v=e v$. We write $v^{v}$ for $v(v)$. For example, $F^{(n)}$ has the standard ghost submodule $\varphi_{\mathbb{0}}^{(n)}$, with

$$
\left(v_{1}, \ldots, v_{n}\right)^{v}=\left(v_{1}^{v}, \ldots, v_{n}^{v}\right) .
$$

Definition 2.9. The tangible vectors of $F^{(n)}$ are those $\left(v_{1}, \ldots, v_{n}\right) \neq 0$ such that each $v_{i} \in \mathscr{T}_{\mathbb{0}}$.

Lemma 2.10. The following properties are satisfied for all $\alpha \in F, v, w \in F^{(n)}$ :

(1) $(\alpha v)^{v}=\alpha v^{v}=\alpha^{v} v$.

(2) $(v+w)^{v}=v^{v}+w^{v}$.

Proof. (1) $(\alpha v)^{v}=e(\alpha v)=(e \alpha) v=(\alpha e) v=\alpha(e v)=\alpha v^{v}$.

(2) $(v+w)^{v}=e(v+w)=e v+e w=v^{v}+w^{v}$. 
As with supertropical semirings, we define the ghost surpassing relation $\models_{\mathrm{gs}}$ for vectors $v=\left(v_{1}, \ldots, v_{n}\right), w=\left(w_{1}, \ldots, w_{n}\right)$ by

$$
v \models_{\mathrm{gs}} w \quad \text { if } \quad v_{i} \models_{\mathrm{gs}} w_{i} \text { for } 1 \leq i \leq n .
$$

We say that two vectors $v, w$ are $v$-matched, written $v \cong_{v} w$, if $v^{v}=w^{v}$. Likewise, we write $v \geq_{v} w$ if $v^{v} \models_{\mathrm{gs}} w^{v}$.

\section{Example 2.11.}

$$
\left(v_{1}, \ldots, v_{n}\right) \geq_{v}\left(w_{1}, \ldots, w_{n}\right)
$$

in $R^{(n)}$ if and only if $v_{i} \geq_{v} w_{i}$ for each $1 \leq i \leq n$.

Also, for elements $v, w$ in $V$, we define

$$
v \Upsilon_{\mathrm{gd}} w \text { if } v+w \in \mathcal{H}_{\mathbb{D}} .
$$

Remark 2.12. (i) If $v \models_{\mathrm{gs}} w$, then $v+w \in \mathscr{H}_{0}$; thus $v \curlyvee_{\mathrm{gd}} w$.

(ii) If $v_{i} \models_{\mathrm{gs}} w$ for $i=1,2$, then $v_{1}+v_{2} \models_{\mathrm{gs}} w$.

Lemma 2.13. The following property holds for all $v, w \in V, h \in \mathscr{H}_{\mathbb{D}}$ :

$$
v=w+h \Longrightarrow v+h=v \text {. }
$$

Proof. $v=w+h=w+h+h=v+h$.

Proposition 2.14. Any vector space $V$ satisfies the following property, for $v, h_{1}$, $h_{2} \in V$ :

$$
v+h_{1}+h_{2}=v \Rightarrow v+h_{2}=v .
$$

Proof. $v=v+h_{1}+h_{2}=\left(v+h_{1}+h_{2}\right)+\left(h_{1}+h_{2}\right)=v+e h_{1}+e h_{2}$. Take $w=v+e h_{1}$ and $h=e h_{2}$ in the lemma to get $v=v+e h_{2}=\left(v+h_{1}+h_{2}\right)+h_{2}=v+h_{2}$.

Corollary 2.15. The ghost surpassing relation on (supertropical) vector spaces is a partial order.

Almost tangible vectors. Since one also has the example of supertropical algebras arising from tropicalizing Puiseux series [Izhakian and Rowen 2010], we digress briefly to discuss the situation when $v$ is not necessarily $1: 1$.

Remark 2.16. Define

$$
\mathscr{T}_{e}:=\left\{a \in \mathcal{T}: a \cong_{\nu} \mathbb{1}_{R}\right\} .
$$

This is a submonoid of $\mathscr{T}$, and in fact $\mathcal{T}_{e} \cup\{e\}$ is a supertropical subsemifield ${ }^{\dagger}$ of $F$.

Lemma 2.17. Generalizing Remark 2.6(i), an element $a \in F$ is tangible if and only if the following condition holds: $a \models_{\mathrm{gs}} b$ implies $b=\alpha$ a for some $\alpha \in \mathscr{T}_{e}$. 
Proof. Remark 2.6(i) yields the forward implication. Conversely, suppose $a$ is not tangible, that is, $a \in \mathscr{G}$, so $a=a^{v}$. Then $a \models_{\mathrm{gs}} \hat{a}$, where $\hat{a} \in \mathcal{T}$ and $(\hat{a})^{v}=a$. The condition implies $\hat{a}=\alpha a$ for some $\alpha \in \mathscr{T}_{e}$, which is impossible since $\alpha a \in \mathscr{G}$.

Motivated by Lemma 2.17, we have an abstract definition of tangibility for any (supertropical) vector space $V$ over a supertropical semifield:

Definition 2.18. The almost tangible vectors of $V$ are those elements $v \in V$ for which $v \models_{\mathrm{gs}} w$, for all $w \in V$, implies $w \in \mathscr{T}_{e} v$.

Remark 2.19. A nonzero ghost vector $v$ cannot be almost tangible. Indeed, we would have $v \models_{\mathrm{gs}} \mathbb{O}$, implying $\mathbb{O} \in \mathscr{T}_{e} v$, a contradiction.

Example 2.20. Clearly, the almost tangible vectors of $F^{(n)}$ all are tangible.

On the other hand, in logarithmic notation, taking $F=D(\mathbb{R})$, if $V$ is the submodule of $F^{(2)}$ spanned by the vectors $v_{1}=\left(1,1^{v}\right)$ and $v_{2}=(0,1)$, then one sees without difficulty that $v_{1}$ is almost tangible in $V$, although not tangible in $F^{(2)}$.

In fact, a subspace of $F^{(n)}$ need not have any tangible vectors at all, as exemplified by the submodule $F\left(1,1^{v}\right)$ of $F^{(2)}$.

Here is a reduction to the case where $v$ is $1: 1$.

Remark 2.21. We define an equivalence on $F^{\dagger}$ via $a \equiv b$ when either $a=b$ or $a, b \in \mathcal{T}$ with $a \cong_{\nu} b$. In other words, two tangible elements are equivalent if and only if they are $v$-matched. Then we could define the supertropical semifield ${ }^{\dagger} F^{\dagger} / \equiv$ to be $(\mathscr{T} / \equiv) \cup \mathscr{G}$. The ghost map $v$ defines a $1: 1$ function from the equivalence classes of $\mathscr{T}$ to $\mathscr{G}$.

\section{Background from matrices}

Any set $S=\left\{v_{1}, \ldots, v_{m}\right\}$ of $m$ row vectors in $F^{(n)}$ corresponds to an $m \times n$ matrix $A(S)$, whose $m$ rows are the vectors of $S$. We call $A(S)$ the matrix of $S$. We denote by $M_{n}(F)$ the monoid of $n \times n$ matrices over $F$, and by $M_{n}\left(\mathscr{G}_{\mathbb{O}}\right)$ the monoid of $n \times n$ ghost matrices.

We recall that the tropical determinant of an $n \times n$ matrix $A=\left(a_{i, j}\right)$ in $M_{n}(F)$ is really the permanent, which we denote as

$$
|A|=\sum_{\pi \in S_{n}} a_{\pi(1), 1} \ldots a_{\pi(n), n} .
$$

Although the equation $|A B|=|A||B|$ fails over the max-plus algebra, the relation $|A B| \models_{\text {gs }}|A||B|$ holds over a supertropical semiring by Theorem 3.5 of [Izhakian and Rowen 2011a], and any matrix satisfies its characteristic polynomial in the sense of Theorem 5.2 of the same work. The tangible roots of this polynomial are precisely the supertropical eigenvalues of $A$, as given in [Izhakian and Rowen 2011a, Theorem 7.10]. 
We say that the matrix $A$ is nonsingular if $|A|$ is tangible (and thus $A$ is quasiinvertible [Izhakian and Rowen 2011a]); otherwise, $|A| \in \mathscr{G}_{\mathbb{0}}$ (that is, $|A| \models_{\mathrm{gs}} \mathbb{O}_{F}$ by Remark 2.5) and we say that $A$ is singular. Although it was shown in [Izhakian and Rowen 2011a] that the product of nonsingular matrices could be singular, we do have the consolation that the product of nonsingular matrices cannot be ghost; see Theorem 3.5 below.

In [Izhakian and Rowen 2011a], we also defined vectors in $F^{(n)}$ to be tropically independent if no linear combination with tangible coefficients is in $\mathscr{H}_{\mathbb{D}}$.

Recall that a quasi-identity matrix is a nonsingular, multiplicatively idempotent matrix ghost-surpassing the identity matrix; therefore its determinant equals $\mathbb{1}_{F}$. Suppose $A=\left(a_{i, j}\right)$, with $|A|$ tangible. In [Izhakian and Rowen 2011b, Theorem 2.8] one defines the matrix

$$
A^{\nabla}:=\frac{\mathbb{1}_{F}}{|A|} \operatorname{adj}(A)
$$

and obtains the quasi-identity matrices

$$
I_{A}=A A^{\nabla}, \quad I_{A}^{\prime}=A^{\nabla} A .
$$

Remark 3.1. Recall some results on nonsingularity and supertropical dependence:

- $A(S)$ has $m$ tropically independent rows (resp. columns) if and only if $A(S)$ has a nonsingular $m \times m$ submatrix [Izhakian and Rowen 2009, Theorem 3.4; 2011a, Corollary 6.6]

- $|A \operatorname{adj}(A)|=|A|^{n}$ [Izhakian and Rowen 2011b, Theorem 4.9].

- $|\operatorname{adj}(A)|=|A|^{n-1}$ [Izhakian and Rowen 2011a]. Thus, $\operatorname{adj}(A)$ is nonsingular if $A$ is nonsingular.

Thus, it is natural to try to understand linear algebra in terms of the supertropical matrix theory of [Izhakian and Rowen 2011a; 2011b].

\section{Annihilators of matrices.}

Definition 3.2. A vector $v \in V:=F^{(n)}$ (written as a column) g-annihilates an $m \times n$ matrix $A$ if $A v \models_{\mathrm{gs}} \mathbb{O}_{V}$ in $V$. Define

$$
\operatorname{Ann}(A)=\left\{v \in V: A v \models_{\mathrm{gs}} \mathbb{O}_{V}\right\}
$$

this is clearly a subspace of $V$.

Accordingly, $\varphi_{\mathbb{0}}^{(n)} \subseteq \operatorname{Ann}(A)$ for any $m \times n$ matrix $A$.

Remark 3.3. (i) The point of this definition is that the vector $v=\left(\beta_{1}, \ldots, \beta_{m}\right)$ g-annihilates $(A(S))^{\mathrm{t}}$, the transpose of the matrix of $S=\left\{w_{1}, \ldots, w_{m}\right\}$, if and only if $\sum_{i=1}^{m} \beta_{i} w_{i} \models_{\mathrm{gs}} \mathbb{O}_{V}$. Thus, tangible g-annihilators correspond to tropical dependence relations. 
(ii) A (nonzero) tangible vector cannot g-annihilate a nonsingular matrix, since the columns are tropically independent.

We can improve this result to include vectors that are not necessarily tangible.

Lemma 3.4. The diagonal of the product $I_{A} I_{B}$ of quasi-identity matrices $I_{A}, I_{B}$ cannot all be ghosts.

Proof. Recall from [Izhakian and Rowen 2011a, §3.2] the weighted digraph $G=$ $(\mathscr{V}, \mathscr{E})$ of an $n \times n$ matrix $A=\left(a_{i, j}\right)$, which is defined to have vertex set $\mathscr{V}=$ $\{1, \ldots, n\}$ and an edge $(i, j)$ from $i$ to $j$ (of weight $a_{i, j}$ ) whenever $a_{i, j} \neq \mathbb{O}_{F}$.

Write $I_{A}=\left(a_{i, j}\right)$ and $I_{B}=\left(b_{i, j}\right)$. If the assertion is false, then for each $i_{t}$, the $i_{t}, i_{t}$ diagonal entry must be a ghost, so there must be $i_{t+1}$ such that $a_{i_{t}}, i_{t+1} b_{i_{t+1}, i_{t}} \geq_{v} \mathbb{1}_{F}$. This means each edge from $i_{t}$ to $i_{t+1}$ in the weighted digraph $G$ of $I_{A} I_{B}$ has weight $\geq_{\nu} 1$. By the pigeonhole principle, the path of vertices of $i_{1}, i_{2}, i_{3}, \ldots, i_{n+1}$ contains a cycle, say from $i_{s}$ to $i_{s}^{\prime}$. But the weight of any nonloop cycle in a quasi-identity has $v$-value $<\mathbb{1}_{F}$. (Otherwise, multiplying by the entries $a_{i, i}$ for all vertices $i$ not in the cycle gives an extra summand $\geq e=\mathbb{1}_{F}^{v}$ for $\left|I_{A}\right|$, contrary to $\left|I_{A}\right|=\mathbb{1}_{F}$.) Hence

$$
\mathbb{1}_{F} \leq_{v} \prod_{k=s}^{s^{\prime}-1} a_{i_{k}, i_{k+1}} b_{i_{k+1}, i_{k}}=\prod_{k=s}^{s^{\prime}-1} a_{i_{k}, i_{k+1}} \prod_{k=s}^{s^{\prime}-1} b_{i_{k+1}, i_{k}}<_{v} \mathbb{1}_{F} \mathbb{1}_{F}=\mathbb{1}_{F},
$$

a contradiction.

Theorem 3.5. The product of two nonsingular $n \times n$ matrices cannot be in $M_{n}\left(\mathscr{G}_{\mathbb{O}}\right)$.

Proof. If $A B$ is ghost for $A, B$ nonsingular, then $\left(A^{\nabla} A\right)\left(B B^{\nabla}\right) \in M_{n}\left(\varphi_{\mathbb{O}}\right)$, contradicting the lemma.

On the other hand, examples were given in [Izhakian and Rowen 2011a] in which the product of two nonsingular $n \times n$ matrices is singular. Here is a related example using quasi-identities:

Example 3.6. The matrices

$$
A=\left(\begin{array}{cc}
0 & 0^{\nu} \\
-\infty & 0
\end{array}\right), \quad B=\left(\begin{array}{cc}
0 & -\infty \\
0^{v} & 0
\end{array}\right)
$$

over $D(\mathbb{R})$ are nonsingular, but $A B=\left(\begin{array}{cc}0^{v} & 0^{v} \\ 0^{v} & 0\end{array}\right)$ and $B A=\left(\begin{array}{cc}0 & 0^{v} \\ 0^{v} & 0^{v}\end{array}\right)$ are singular.

\section{Tropical dependence}

Dependence plays a major role in module theory. The familiar definition becomes degenerate for supertropical vector spaces; the following modification from [Izhakian and Rowen 2011a], in which the role of zero is replaced by the ghost ideal, is more suitable for our purposes. 
Definition 4.1. Suppose $V$ is a supertropical vector space over $F$. A family of elements $S=\left\{w_{i}: i \in I\right\} \subset V$ is tropically dependent if there exists a nonempty finite subset $I^{\prime} \subset I$ and a family $\left\{\alpha_{i}: i \in I^{\prime}\right\} \subset \mathcal{T}$ such that

$$
\sum_{i \in I^{\prime}} \alpha_{i} w_{i} \in \mathscr{H}_{\mathbb{D}} .
$$

Any such relation (4-1) is called a tropical dependence for $S$. A subset $S \subset V$ is called tropically independent if it is not tropically dependent.

Given an element $v \in V$, we say that $v$ is tropically dependent on a family $S=\left\{w_{i}: i \in I\right\}$ if $S \cup\{v\}$ is tropically dependent, in which case we write $v \curlyvee_{\mathrm{gd}} S$. (In particular, $v \curlyvee_{\mathrm{gd}}\{v\}$.) A subset $S^{\prime}$ of $V$ is tropically dependent on $S$ if $v \Upsilon_{\mathrm{gd}} S$ for each $v \in S^{\prime}$.

An easy observation:

Remark 4.2. Suppose $S=\left\{w_{i}: i \in I\right\} \subset V$. For any given set $\left\{\alpha_{i}: i \in I\right\} \subset \mathcal{T}$ of tangible elements of $F$, the set $S$ is tropically independent if and only if $\left\{\alpha_{i} w_{i}: i \in I\right\}$ is tropically independent.

Lemma 4.3. If a set $S^{\prime}$ is tropically dependent on $S \subset F^{(n)}$, then the supertropical vector space $V$ of $F^{(n)}$ spanned by $S^{\prime}$ is also tropically dependent on $S$.

Proof. It is enough to show that if $v, v^{\prime} \in F^{(n)}$ are tropically dependent on $S$, then $v+v^{\prime}$ is also tropically dependent on $S$. Write $v+\sum \alpha_{i} w_{i} \in \mathscr{H}$ and $v^{\prime}+\sum \alpha_{i}^{\prime} w_{i} \in \mathcal{H}$ for $w_{i} \in S$. It is enough to check this on each component. Thus, we may assume that $v, v^{\prime}, w_{i}$, and $w_{i}^{\prime}$ are all in $F$. If $v \cong_{v} v^{\prime}$, then $v+v^{\prime} \in \mathscr{G}$. On the other hand, if $v>_{v} v^{\prime}$, then $v+v^{\prime}=v$ is already tropically dependent on $S$, so the assertion is clear.

\section{Tropical d-bases and rank.}

Definition 4.4. A $d$-base (for dependence base) of a supertropical vector space $V$ is a maximal set of tropically independent elements of $V$. The rank of a d-base $\mathscr{B}$, denoted $\operatorname{rk}(\mathscr{B})$, is the number of elements of $\mathscr{B}$.

Our d-base corresponds to the "basis" in [Maclagan and Sturmfels 2009, Definition 5.2.4].

Proposition 4.5. Any subspace of $F^{(n)}$ is tropically dependent on any subset $S$ of $n$ tropically independent elements. All d-bases of $F^{(n)}$ have precisely $n$ elements.

Proof. By Theorem 6.6 of [Izhakian and Rowen 2011a], the matrix $A$ of $S$ is nonsingular if and only if $S$ is tropically independent, so in particular any d-base $\mathscr{B}$ of $F^{(n)}$ must have at least $n$ elements. Also recall that any $n+1$ vectors of $F^{(n)}$ are tropically dependent, by Corollary 6.7 of the same work, so $\mathscr{B}$ has precisely $n$ elements. 
This leads us to the following definition.

Definition 4.6. The rank of a supertropical vector space $V$ is defined as

$$
\operatorname{rk}(V):=\max \{\operatorname{rk}(\mathscr{B}): \mathscr{B} \text { is a d-base of } V\} .
$$

For $V \subseteq F^{(n)}$, the tangible rank of $V$ is defined as

$$
\mathrm{t}-\mathrm{rk}(V):=\max \left\{\operatorname{rk}(\mathscr{B}): \mathscr{B} \subset \mathscr{T}_{\mathbb{O}}^{(n)} \text { is a d-base of } V\right\} .
$$

We have just seen that $\mathrm{t}-\mathrm{rk}\left(F^{(n)}\right)=\operatorname{rk}\left(F^{(n)}\right)=n$.

Corollary 4.7. If $V \subset F^{(n)}$, then $\operatorname{rk}(V) \leq n$.

Proof. Any d-base of $V$ is contained in a d-base of $F^{(n)}$ whose order must be that of the standard base given in (5-1) below, which is $n$.

We might have liked $\operatorname{rk}(V)$ to be independent of the choice of d-base of $V$, for any supertropical vector space $V$. This is proved in the classical theory of vector spaces by showing that dependence is transitive. However, transitivity fails in the supertropical theory, since we have the following sort of counterexample.

Example 4.8. In logarithmic notation, over $D(\mathbb{R})^{(3)}$, the vector $v=(0,1,3)$ is tropically dependent on $W=\left\{w_{1}, w_{2}\right\}$, where $w_{1}=(1,1,2)$ and $w_{2}=(1,1,3)$, since $v+w_{1}+w_{2}=\left(1^{v}, 1^{v}, 3^{v}\right)$. Furthermore, $W$ is tropically dependent on $U=\left\{u_{1}, u_{2}\right\}$, where $u_{1}=(1,1,0)$ and $u_{2}=(-\infty,-\infty, 1)$, since

$$
w_{1}+u_{1}+1 u_{2}=\left(1^{v}, 1^{v}, 2^{v}\right), \quad w_{2}+u_{1}+2 u_{2}=\left(1^{v}, 1^{v}, 3^{v}\right) .
$$

But $v, u_{1}$, and $u_{2}$ are tropically independent, since the tropical determinant of the matrix whose rows are these vectors is $3 \in \mathcal{T}$.

In fact, different d-bases may contain different numbers of elements, even when tangible. An example is given in [Maclagan and Sturmfels 2009, Example 5.4.20], which is reproduced here with different entries.

Example 4.9. Consider the following vectors in $D(\mathbb{R})^{(3)}$ :

$$
v_{1}=(5,5,0), \quad v_{2}=(5,5,4), \quad v_{3}=(0,1,4), \quad v_{4}=(0,2,4) .
$$

Then $v_{1}, v_{2}$, and $v_{3}$ are tropically dependent (since their sum $\left(5^{v}, 5^{v}, 4^{v}\right)$ is ghost), and likewise $v_{1}, v_{2}$, and $v_{4}$ are tropically dependent. It follows that $\left\{v_{1}, v_{2}\right\}$ is a d-base for the supertropical vector space $V$ spanned by $v_{1}, v_{2}, v_{3}$, and $v_{4}$. But $v_{2}, v_{3}$, and $v_{4}$ are tropically independent since their determinant is 11 , which is tangible; hence, $\left\{v_{2}, v_{3}, v_{4}\right\}$ is also a d-base of $V$.

We do have a consolation. 
Lemma 4.10. If the vectors $v_{1}, \ldots, v_{k} \in F^{(n)}$ are tropically independent and the vector $v$ is tangible, then there are $i_{1}, \ldots, i_{k-1}$ in $\{1, \ldots, k\}$ such that the vectors $v_{i_{1}}, \ldots, v_{i_{k-1}}, v$ are tropically independent.

Proof. Let $A$ be the $(k+1) \times n$ matrix whose rows are $v_{1}, \ldots, v_{k}, v$, and let $A_{0}$ denote the $k \times n$ matrix of the first $k$ rows $v_{1}, \ldots, v_{k}$. Then $A_{0}$ has a nonsingular $k \times k$ submatrix obtained by deleting $n-k$ columns; deleting these columns in $A$, we have reduced to the case that $n=k$, that is, $A$ is a $(k+1) \times k$ matrix. Now let $A_{0}^{\prime}=\left(a_{j, i}^{\prime}\right)=\operatorname{adj}\left(A_{0}\right)$, which is nonsingular; see Remark 3.1. We are done unless for each row $i \leq k$, the $k \times k$ submatrix of $A$ obtained by deleting the $i$ row is singular, which means that $\sum_{j=1}^{k} a_{i, j}^{\prime} a_{k+1, j}$ is ghost. This means that the vector $\left(a_{k+1,1}, \ldots, a_{k+1, k}\right) \mathrm{g}$-annihilates the nonsingular matrix $A_{0}^{\prime}$, which is impossible.

Proposition 4.11. For any tropical subspace $V$ of $F^{(n)}$ and any tangible $v \in V$, there is a tangible d-base of $V$ containing $v$ whose rank is that of $V$.

Proof. Take a tangible d-base of $V$ of maximal rank, and apply the lemma.

Example 4.12 (failure of the analog of Proposition 4.11 for nontangible vectors). Consider the supertropical vector space $W \subset D(\mathbb{R})^{(2)}$ spanned by $w_{1}=(0,1)$ and $w_{2}=(0,2)$. Then $v=\left(1,3^{v}\right)$ comprises a d-base of $W$, consisting of only one element.

Proposition 4.13. If $A$ is a matrix of rank $m$, its $g$-annihilator has a tangible tropically independent set of rank $\geq n-m$.

Proof. Take $m$ tropically independent rows $v_{1}, \ldots, v_{m}$ of $A$, which we may assume are the first $m$ rows of $A$. For any other row $v_{u}$ of $A(m<u \leq n)$, we have $\beta_{u, 1}, \ldots, \beta_{u, m} \in \mathscr{T}_{\mathbb{O}}$ such that $v_{u}+\sum \beta_{i, j} v_{i} \in \mathscr{G}_{\mathbb{0}}^{(n)}$. Letting $B$ be the $(n-m) \times n$ matrix whose $(i, j)$ entries are $\beta_{i, j}$ for $1 \leq i, j \leq m$, and for which $\beta_{i, j}=\delta_{i, j}$ (the Kronecker delta) for $m<j \leq n$, we see that $B$ contains an $(n-m) \times(n-m)$ identity submatrix and so has tangible rank $\geq n-m$, but $B A$ is ghost.

Example 4.14. We exhibit a $3 \times 3$ matrix $A$ over $D(\mathbb{R})$ of rank $m=2$, all of whose entries are tangible, although $\operatorname{rk}(\operatorname{Ann}(A))=2>3-2$. Take

$$
A=\left(\begin{array}{lll}
4 & 4 & 0 \\
4 & 4 & 1 \\
4 & 4 & 2
\end{array}\right) .
$$

$A$ is g-annihilated by the tropically independent vectors $v_{1}=(1,1,0)^{\mathrm{t}}$ and $v_{2}=$ $(1,1,1)^{\mathrm{t}}$, since $A v_{1}=A v_{2}=(5,5,5)^{\mathrm{t}}$.

Note that this kind of example requires $n \geq 3$, in view of Theorem 3.5. 
Saturated dependence relations. Let us study tropical dependence relations in $F^{(n)}$ more closely. Example 5.7(ii) below shows that a tropical dependence of a vector $v$ on an independent set $S=\left\{w_{i}: i \in I\right\}$ is not uniquely determined. Nevertheless, in this subsection we do get a "canonical" tropical dependence relation, which we call saturated. But first, in order for tropical dependence relations to be well-defined with respect to the ghost map $v: F \rightarrow \mathscr{G}_{\mathbb{0}}$, we verify the following condition.

Lemma 4.15. Any subspace of $F^{(n)}$ (with $\mathscr{H}_{\mathbb{0}}=\mathscr{G}_{\mathbb{0}}^{(n)}$ ) satisfies the property that whenever $\alpha_{i}, \beta_{i} \in \mathcal{T}$ with $\alpha_{i} \cong{ }_{\nu} \beta_{i}$,

$$
\sum_{i} \alpha_{i} w_{i} \in \mathscr{H}_{\mathbb{0}} \Longleftrightarrow \sum_{i} \beta_{i} w_{i} \in \mathscr{H}_{\mathbb{0}} \text {. }
$$

Proof. The argument is analogous to that of Lemma 4.3. The condition clearly passes to submodules, so it is enough to prove it for $F^{(n)}$, and thus to check (4-2) on each component. We write $w_{i, j}$ for the $j$-component of $w_{i}$. Note that $\alpha_{i} w_{i, j} \cong_{v} \beta_{i} w_{i, j}$ for each $i$. There are two ways for $\sum_{i} \alpha_{i} w_{i, j} \in \mathscr{G}_{\mathbb{0}}$ :

(1) Some $\alpha_{i^{\prime}} w_{i^{\prime}, j}$ dominates $\sum_{i} \alpha_{i} w_{i, j}$ and is ghost, implying $w_{i^{\prime}, j} \in \mathscr{G}_{\mathbb{0}}$, so

$$
\sum_{i} \beta_{i} w_{i, j}=\beta_{i^{\prime}} w_{i^{\prime}, j}=\alpha_{i^{\prime}} w_{i^{\prime}, j} \in \mathscr{G}_{\mathbb{O}}
$$

(2) Two essential summands $\alpha_{i^{\prime}} w_{i^{\prime}, j}$ and $\alpha_{i^{\prime \prime}} w_{i^{\prime \prime}, j}$ are $\nu$-matched. But then

$$
\begin{aligned}
\sum_{i} \beta_{i} w_{i, j} & =\beta_{i^{\prime}} w_{i^{\prime}, j}+\beta_{i^{\prime \prime}} w_{i^{\prime \prime}, j}=\left(\beta_{i^{\prime}} w_{i^{\prime}, j}\right)^{v} \\
& =\left(\alpha_{i^{\prime}} w_{i^{\prime}, j}\right)^{v}=\alpha_{i^{\prime}} w_{i^{\prime}, j}+\alpha_{i^{\prime \prime}} w_{i^{\prime \prime}, j}=\sum_{i} \alpha_{i} w_{i, j} \in \mathscr{G}_{\mathbb{0}}
\end{aligned}
$$

We examine the tropical dependence

$$
v \curlyvee_{\mathrm{gd}} \sum_{i \in I} \alpha_{i} w_{i}
$$

Lemma 4.16. Suppose $V=F^{(n)}$. If $v \curlyvee_{\mathrm{gd}} \sum_{i \in I} \alpha_{i} w_{i}$ and $v \curlyvee_{\mathrm{gd}} \sum_{i \in I} \beta_{i} w_{i}$ for $\alpha_{i}, \beta_{i} \in \mathscr{T}_{\mathbb{D}}$, then taking $\gamma_{i}=\widehat{\alpha_{i}+\beta_{i}}$, we have

$$
v \curlyvee_{\mathrm{gd}} \sum_{i \in I} \gamma_{i} w_{i}
$$

Proof. Checking each component in turn, we may assume that $V=F$. We proceed as in Lemma 4.15. Namely, $v \curlyvee_{\mathrm{gd}} \sum_{i \in I} \alpha_{i} w_{i}$ (resp. $v \curlyvee_{\mathrm{gd}} \sum_{i \in I} \beta_{i} w_{i}$ ) implies one of the following:

(1) $v$ and some term $\alpha_{i^{\prime}} w_{i^{\prime}}$ dominate (resp. $v$ and $\beta_{i^{\prime}} w_{i^{\prime}}$ dominate), in which case $\gamma_{i^{\prime}}=\alpha_{i^{\prime}}\left(\operatorname{resp} \cdot \gamma_{i^{\prime}}=\beta_{i^{\prime}}\right)$. 
(2) $\alpha_{i^{\prime}} w_{i^{\prime}}$ and $\alpha_{i^{\prime \prime}} w_{i^{\prime \prime}}$ dominate (resp. $\beta_{i^{\prime}} w_{i^{\prime}}$ and $\beta_{i^{\prime \prime}} w_{i^{\prime \prime}}$ dominate), in which case $\gamma_{i^{\prime}}=\alpha_{i^{\prime}}$ and $\gamma_{i^{\prime \prime}}=\alpha_{i^{\prime \prime}}\left(\right.$ resp. $\gamma_{i^{\prime}}=\beta_{i^{\prime}}$ and $\left.\gamma_{i^{\prime \prime}}=\beta_{i^{\prime \prime}}\right)$.

(3) Some ghost term $\alpha_{i^{\prime}} w_{i^{\prime}}\left(\right.$ resp. $\beta_{i^{\prime}} w_{i^{\prime}}$ ) dominates, in which case $\gamma_{i^{\prime}}=\alpha_{i^{\prime}}$ (resp. $\left.\gamma_{i^{\prime}}=\beta_{i^{\prime}}\right)$.

Lemma 4.16 gives us a partial order on the coefficients of the tropical dependence relations of $v$ on a set $S$, and motivates the following definition:

Definition 4.17. We say that the support of a tropical dependence

$$
\alpha v \curlyvee_{\mathrm{gd}} \sum_{i \in I} \alpha_{i} w_{i}
$$

(where $\alpha \in \mathscr{T}$ and $\alpha_{i} \in \mathscr{T}_{\mathbb{O}}$ ) is the set

$$
\left\{i \in I: \alpha_{i} \neq \mathbb{O}_{F}\right\} \text {. }
$$

A tropical dependence of minimal support is called irredundant.

A vector does not have a unique tropical dependence on a d-base. For example, if each entry of a vector $w_{1}$ is nonzero and $\alpha \in F$ has a suitably low $\nu$-value, then $w_{1}+\alpha w_{2}=w_{1}$. We do have a slight consolation.

Lemma 4.18. If $\sum_{i=1}^{k} \alpha_{i} w_{i} \curlyvee_{\mathrm{gd}} \sum_{i=1}^{k} \beta_{i} w_{i}$ for independent vectors $w_{1}, \ldots, w_{k}$ and tangible $\alpha_{i}, \beta_{i}$, then $\alpha_{i} \cong_{v} \beta_{i}$ for some $i$.

Proof. $\sum_{i=1}^{k}\left(\alpha_{i}+\beta_{i}\right) w_{i} \in \mathscr{H}_{\mathbb{\bigotimes}}$, implying that not all $\alpha_{i}+\beta_{i}$ are tangible, so that $\alpha_{i} \cong{ }_{\nu} \beta_{i}$.

We can do better. A tropical dependence of $v$ on a tropically independent set $S$ is called saturated if the coefficients $\alpha_{i}$ in (4-3) all are maximal possible with respect to $\geq_{\nu}$; in other words, whenever $v+\sum_{i=1}^{l} \beta_{i} w_{i} \in \mathscr{G}_{\mathbb{0}}^{(n)}$ with $\beta_{i} \in \mathscr{T}_{\mathbb{D}}$, then each $\beta_{i} \leq_{v} \alpha_{i}$.

Remark 4.19. If

$$
v \curlyvee_{\mathrm{gd}} \sum_{i=1}^{l} \alpha_{i} w_{i}
$$

is a saturated tropical dependence, then for any $k \leq l$ and for $v^{\prime}=v+\sum_{i=1}^{k} \alpha_{i} w_{i}$,

$$
v^{\prime} \Upsilon_{\mathrm{gd}} \sum_{i=k+1}^{l} \alpha_{i} w_{i}
$$

is also a saturated tropical dependence, since any $v$-larger tropical dependence for (4-5) would yield the corresponding $v$-larger tropical dependence for (4-4). 
Theorem 4.20. Any irredundant tropical dependence

$$
v \Upsilon_{\mathrm{gd}} \sum_{i=1}^{l} \alpha_{i} w_{i}
$$

can be extended to a unique (up to equivalence in the sense of Remark 2.21) saturated tropical dependence of $v$ on $S=\left\{w_{1}, \ldots, w_{l}\right\}$, having the same support.

Remark 4.21. When the vector $v$ is tangible and $S$ is a d-base, Theorem 4.20 is an immediate consequence of [Izhakian and Rowen 2011b, Theorems 3.5 and 3.8], which shows that $A x \models_{\mathrm{gs}} v$ has the maximal tangible vector solution $x=\widehat{v}\left(A^{\nabla} v\right)$, where $A^{\nabla}=(1 /|A|) \operatorname{adj}(A)$. Here we take $A$ to be the matrix of $S$, which is nonsingular, and $x$ to be the vector $\left(\alpha_{1}, \ldots, \alpha_{l}\right)^{\mathrm{t}}$.

In general, $x=A^{\nabla} v$ is a solution for the matrix equation $A x \models_{\mathrm{gs}} v$, which, when $v$ is written as a row, is $x A^{\mathrm{t}} \models_{\mathrm{gs}} v$. (In a sense, row form is more natural, since the matrix of $S$ is obtained from the rows.) But this solution $x$ need not be tangible.

Here is a direct combinatoric proof of Theorem 4.20 that does not rely on matrix theory, and does not depend on the additional assumption of tangibility of $S$.

Proof of Theorem 4.20. Uniqueness of a saturated tangible solution is obvious, since one could just take the sup of any two distinct saturated tropical dependences to get a contradiction. This also gives the motivation for proving existence. Write $v=\left(v_{1}, \ldots, v_{n}\right)$. We start with some tropical dependence (4-6), which need not be saturated, with the aim of checking whether we can modify it until it is saturated. In principle, we could increase the $\nu$-values of the coefficient $\alpha_{i}$ if at each component $j$ of the vector $\alpha_{i} w_{i}$ the $v$-value of $v_{j}$ is not attained, and this is the main idea behind the proof. But increasing $\alpha_{i}$ still may not yield a saturated tropical dependence, since the coefficient may be allowed to increase further, so long as some other term in the tropical dependence also is adjusted so as to have a $j$-component of the same $v$-value. Since these $j$-components are the most difficult to keep track of, we pay special attention to them. Write $w_{i, j}$ for the $j$-component of $w_{i}$.

We say that an index $j \leq n$ has type 1 if $v_{j}$ is not dominated by $\sum_{i} \alpha_{i} w_{i, j}$, which means that either $v_{j}$ itself is ghost, or else there is precisely one $i$ with $\alpha_{i} w_{i, j}$ matching $v_{j}$, and this $w_{i, j} \in \mathscr{T}$.

We say that $j$ has type 2 for $v$ if $v_{j}$ is dominated by $\sum_{i} \alpha_{i} w_{i, j}$, which means that either there exists $i$ such that $\alpha_{i} w_{i, j}$ is ghost and dominates $v_{j}$ or there are $i, i^{\prime}$ such that $\alpha_{i} w_{i, j}$ and $\alpha_{i^{\prime}} w_{i^{\prime}, j}$ are $v$-matched and both dominate $v_{j}$.

Note that increasing the coefficients $\alpha_{i}$ in a tropical dependence cannot change the type of an index $j$ from type 2 to type 1. Also, at least one index must have type 1 , since otherwise $\sum \alpha_{i} w_{i, j} \in \mathscr{G}_{\mathbb{0}}^{(n)}$, contrary to the hypothesis that the $w_{i}$ are tropically independent. We choose our tropical dependence such that the number of indices of type 1 is minimal. In this case, if $\alpha_{i} w_{i, j} \nu$-matches $v_{j}$ for $j$ of type 1 , 
we cannot find a $\nu$-greater tropical dependence in which $\alpha_{i}$ is increased, since this would force the tropical dependence to have an extra type 2 index. Thus, in this case we say $w_{i}$ is anchored at $j$. Renumbering the vectors, we may assume that $w_{1}, \ldots, w_{k}$ are anchored at various indices, and replace $v$ by $v^{\prime}=v+\sum_{i=1}^{k} \alpha_{i} w_{i}$. Now we have a new tropical dependence

$$
v^{\prime}+\sum_{i=k+1}^{l} \alpha_{i} w_{i} \in \varphi_{0}^{(n)},
$$

which by induction on $l$ can be extended to a saturated tropical dependence

$$
v^{\prime} \curlyvee_{\mathrm{gd}} \sum_{i=k+1}^{l} \alpha_{i}^{\prime} w_{i}
$$

But then the tropical dependence

$$
v \Upsilon_{\mathrm{gd}}\left(\sum_{i=1}^{k} \alpha_{i} w_{i}+\sum_{i=k+1}^{l} \alpha_{i}^{\prime} w_{i}\right)
$$

is saturated, since $w_{1}, \ldots, w_{k}$ are anchored.

Proposition 4.22. If

$$
v \curlyvee_{\mathrm{gd}} \sum_{i=1}^{l} \alpha_{i} w_{i} \quad \text { and } \quad v^{\prime} \curlyvee_{\mathrm{gd}} \sum_{i=1}^{l} \alpha_{i}^{\prime} w_{i}
$$

are saturated tropical dependences, then

$$
\left(v+v^{\prime}\right) \curlyvee_{\mathrm{gd}} \sum_{i=1}^{l}\left(\widehat{\left(\alpha_{i}+\alpha_{i}^{\prime}\right.}\right) w_{i}
$$

is also a saturated tropical dependence.

Proof. Again we have two proofs, the first using results from [Izhakian and Rowen $2011 \mathrm{~b}$ ] in the case when $v, v^{\prime}$ are tangible and the matrix $A$ of the $w_{i}$ is nonsingular. In the first case, one just takes the solutions $x=\widehat{A^{\nabla} v}$ and $x^{\prime}=\widehat{A^{\nabla} v^{\prime}}$ for the vectors accompanying the $\alpha_{i}$ and the $\alpha_{i}^{\prime}$, and then notes that

$$
\widehat{v}\left(A^{\nabla} v+A^{\nabla} v^{\prime}\right)=\widehat{v}\left(A^{\nabla}\left(v+v^{\prime}\right)\right) .
$$

For the general case, one needs to modify the second proof of Theorem 4.20 for the vector $v+v^{\prime}$. Namely, consider the tropical dependence

$$
\left(v+v^{\prime}\right) \curlyvee_{\mathrm{gd}} \sum_{i=1}^{l} \gamma_{i} w_{i},
$$

where $\gamma_{i}=\left(\widehat{\alpha_{i}+\alpha_{i}^{\prime}}\right)$. At least one index in this tropical dependence must have type 1 
for $v+v^{\prime}$, since otherwise the $w_{i}$ are tropically dependent. We choose our tropical dependence such that the number of indices of type 1 is minimal. As before, if $\gamma_{i} w_{i, j}$ $v$-matches $v_{j}$ for $j$ of type 1, we cannot find a larger tropical dependence in which $\gamma_{i}$ is increased, so $w_{i}$ is anchored at $j$. Again, we may assume that $w_{1}, \ldots, w_{k}$ are anchored at various indices, and replace $v+v^{\prime}$ by $v^{\prime \prime}=v+v^{\prime}+\sum_{i=1}^{k} \gamma_{i} w_{i}$. But

$$
\left(v+\sum_{i=1}^{k} \alpha_{i} w_{i}\right) \curlyvee_{\mathrm{gd}} \sum_{i=k+1}^{l} \alpha_{i} w_{i} \quad \text { and } \quad\left(v^{\prime}+\sum_{i=1}^{k} \alpha_{i}^{\prime} w_{i}\right) \curlyvee_{\mathrm{gd}} \sum_{i=k+1}^{l} \alpha_{i}^{\prime} w_{i}
$$

are saturated tropical dependences by Remark 4.19, so by induction on $l$,

$$
v^{\prime \prime} \curlyvee_{\mathrm{gd}} \sum_{i=k+1}^{l} \gamma_{i} w_{i}
$$

is a saturated tropical dependence. But then the tropical dependence

$$
v \gamma_{\mathrm{gd}}\left(\sum_{i=1}^{k} \gamma_{i} w_{i}+\sum_{i=k+1}^{l} \gamma_{i} w_{i}\right)
$$

is saturated.

\section{Tropical spanning}

In this section, we consider further the fundamental question of what "base" should mean for supertropical vector spaces. The d-base (defined above) competes with another notion to be obtained from $\models_{\text {gs }}$. But for the moment we turn to the naive analog from the classical theory of linear algebra.

Definition 5.1 (classical bases and the standard base). A (supertropical) vector space $V$ over a semifield $F$ is classically spanned by a set $S=\left\{w_{i}: i \in I\right\}$ if every element of $V$ can be written in the form

$$
v=\sum_{i \in J} \alpha_{i} w_{i}
$$

for $\alpha_{i} \in F$ and some finite index set $J \subset I$.

A set $\mathscr{B}=\left\{b_{1}, \ldots, b_{n}\right\} \subset V$ is a classical base of a vector space $V$ over a semifield $F$ if every element of $V$ can be written uniquely in the form $\sum_{i=1}^{n} \alpha_{i} b_{i}$ for $\alpha_{i} \in F$.

The standard base of $F^{(n)}$ is the classical base defined as

$$
\begin{aligned}
\varepsilon_{1} & =\left(\mathbb{1}_{F}, \mathbb{O}_{F}, \ldots, \mathbb{O}_{F}\right), \\
\varepsilon_{2} & =\left(\mathbb{O}_{F}, \mathbb{1}_{F}, \mathbb{O}_{F}, \ldots, \mathbb{O}_{F}\right), \\
& \ldots \\
\varepsilon_{n} & =\left(\mathbb{O}_{F}, \mathbb{O}_{F}, \ldots, \mathbb{1}_{F}\right) .
\end{aligned}
$$


Proposition 5.2. If $V$ has a classical base $b_{1}, \ldots, b_{n}$, then $V$ is isomorphic to $F^{(n)}$. The proof is standard; one defines the isomorphism $F^{(n)} \rightarrow V$ by

$$
\left(\alpha_{1}, \ldots, \alpha_{n}\right) \mapsto \sum_{j=1}^{n} \alpha_{j} b_{j}
$$

Definition 5.3 (tropical spanning). A vector $v \in V$ is tropically spanned by a set $S=\left\{w_{i}: i \in I\right\} \subset V$ if there exist a nonempty finite subset $J \subset I$ and a family $\left\{\alpha_{i}: i \in J\right\} \subset \mathscr{T}$ such that

$$
v \models_{\mathrm{gs}} \sum_{i \in J} \alpha_{i} w_{i}
$$

In this case, we write $v \models_{\mathrm{gS}} S$.

A subset $S^{\prime} \subseteq V$ is tropically spanned by $S$, written $S^{\prime} \models_{\mathrm{gs}} S$, if $v \models_{\mathrm{gs}} S$ for each $v \in S^{\prime}$.

Remark 5.4 (transitivity for tropical spanning). If $V \models_{\mathrm{gs}} W$ and $W \models_{\mathrm{gs}} U$, then $V \models_{\mathrm{gs}} U$.

Obviously, any set classically spanned by $S$ is tropically spanned; surprisingly, the converse often holds.

Remark 5.5. (i) If a tangible vector $v \in F^{(n)}$ is tropically spanned by a set $S \subset V$, then $v$ is classically spanned by $S$ with the same coefficients, as seen by checking components.

(ii) The assertion in (i) can fail for nontangible $v \in F^{(2)}$. Take $S=\left\{\left(\mathbb{1}_{F}, \mathbb{1}_{F}\right)\right\}$; then $v=\left(\mathbb{1}_{F}, \mathbb{1}_{F}^{v}\right)$ is tropically spanned by $S$ since $\left(\mathbb{1}_{F}, \mathbb{1}_{F}^{v}\right) \models_{\mathrm{gs}}\left(\mathbb{1}_{F}, \mathbb{1}_{F}^{v}\right)$, but is not classically spanned by $S$.

(iii) If $V$ has a classical spanning set $\mathscr{B}$ of almost tangible vectors, and $\mathscr{B}$ is tropically spanned by a set $S$, then $V$ is classically spanned by $S$, by (ii) and transitivity. In particular, if $F^{(n)}$ is tropically spanned by a set $S$, then $F^{(n)}$ is classically spanned by $S$, since $F^{(n)}$ has the standard base.

(iv) Any element tropically spanned by $S$ is also tropically dependent on $S$, but not conversely; for example $v=\left(\mathbb{1}_{F}, \mathbb{1}_{F}\right) \in F^{(2)}$ is tropically dependent on $S=\left\{\left(\mathbb{1}_{F}, \mathbb{1}_{F}^{v}\right)\right\} \subset F^{(2)}$, but $v$ is not tropically spanned by $S$. This leads to an interesting dichotomy to be studied shortly.

(v) Tropical spanning does not satisfy the assertion analogous to Lemma 4.16. For example, take

$$
\left\{w_{1}=(1,2), w_{2}=(1,3)\right\} \subset D(\mathbb{R})^{(2)}
$$

and the vector $v=\left(1,3^{v}\right)$; then $v \models_{\mathrm{gs}} w_{1}$ and $v \models_{\mathrm{gs}} w_{2}$, but $v \not \models_{\mathrm{gs}} w_{1}+w_{2}=$ $\left(1^{v}, 3\right)$. 
Thus, we see that almost tangible vectors already begin to play a special role in the theory of tropical dependence, and could be used instead of tangible vectors in the general theory of supertropical vector spaces.

Lemma 5.6. $W=\left\{v \in V: v \models_{\mathrm{gs}} S\right\}$ is a subspace of $V$ @ for any $S \subset V$.

Proof. If $v=\sum_{i \in I} \alpha_{i} w_{i}+y$ and $v^{\prime}=\sum_{i \in I} \alpha_{i}^{\prime} w_{i}+z$, where $\alpha_{i}, \alpha_{i}^{\prime} \in \mathscr{T}, w_{i} \in S$ and $y, z \in \mathscr{H}_{\mathbb{D}}$, then letting $J=\left\{i: \alpha_{i} \cong_{\nu} \alpha_{i}^{\prime}\right\}$, we have, by bipotence,

$$
v+v^{\prime}=\sum_{i \notin J} \beta_{i} w_{i}+\sum_{i \in J} \alpha_{i}^{v} w_{i}+(y+z) \models_{\mathrm{gs}} \sum_{i \notin J} \beta_{i} w_{i},
$$

where $\beta_{i} \in\left\{\alpha_{i}, \alpha_{i}^{\prime}\right\} \subset \mathscr{T}$. The other verifications are easier.

We call $W$ (in Lemma 5.6) the subspace tropically spanned by $S$, and say that $S$ is a tropically spanning set of $W$.

A supertropical vector space is finitely spanned if it has a finite tropically spanning set.

Example 5.7. Take $R=D(\mathbb{R})$, with logarithmic notation.

(i) The vectors

$$
v_{1}=(1,0,1), \quad v_{2}=(1,1,0), \quad \text { and } \quad v_{3}=(0,1,1)
$$

are tropically dependent in $D(\mathbb{R})^{(3)}$ since their sum is $\left(1^{v}, 1^{v}, 1^{v}\right)$. None of these vectors is tropically spanned by the two other vectors.

(ii) Even when a vector is classically spanned by tropically independent vectors, the coefficients need not be unique. For example,

$$
(4,5)=2(1,1)+2(2,3)=1(1,1)+2(2,3) .
$$

The point of this example is that the first coefficient is sufficiently small so as not to affect the outcome.

(iii) Another such example: The vectors

$$
v_{1}=(-\infty,-\infty, 1), \quad v_{2}=(1,1,-\infty), \quad \text { and } \quad v_{3}=(-\infty, 1,1)
$$

are tropically independent, though classical spanning with respect to them (and thus also tropical spanning) is not unique; e.g., $(3,3,1)=2 v_{2}+v_{3}=v_{1}+2 v_{2}$.

(iv) Another such example: Consider the vectors

$$
v_{1}=(1,4,3), \quad v_{2}=(2,3,4), \quad \text { and } \quad v_{3}=(0,20,20) \text {. }
$$

Then $(3,20,20)=1 v_{2}+v_{3}=2 v_{1}+v_{3}$. 
For $S=\left\{w_{1}, \ldots, w_{n}\right\}$, there need not be a $\nu$-maximal set of $\alpha_{1}, \ldots, \alpha_{l} \in \mathcal{T}$ such that $v \models_{\mathrm{gs}} \sum_{i=1}^{l} \alpha_{i} w_{i}$. For example, in logarithmic notation, take

$$
v=(1,1), \quad w_{1}=(1,0), \quad \text { and } \quad w_{2}=(1,1) .
$$

Then $v=\alpha w_{1}+w_{2}$ for all $\alpha<0$, but taking $\alpha=0$ yields $w_{1}+w_{2}=\left(1^{v}, 1\right)$.

Proposition 5.8. For any subspace $V$ of $F^{(n)}$, the number of elements of any tropically spanning set $S$ of $V$ is at least $\operatorname{rk}(V)$.

Proof. Take a d-base $\left\{v_{1}, \ldots, v_{m}\right\}$ of $V$, where $m=\operatorname{rk}(V) \leq n$. By [Izhakian and Rowen 2009, Theorem 3.4], the $m \times n$ matrix whose rows are $v_{1}, \ldots, v_{m}$ has rank $m$. Taking a nonsingular $m \times m$ submatrix and erasing all the $n-m$ columns not appearing in this submatrix, we may assume that $m=n$ (since we still have a supertropically generating set which we can shrink to a minimal one).

Writing $v_{i} \models_{\mathrm{gs}} \sum \alpha_{i, j} s_{j}$ for suitable $s_{j} \in S$, we can procure a nonsingular matrix whose rows are various $s_{j}$, implying that some subset of $m$ vectors of $S$ is tropically independent, and thus $|S| \geq m$.

We are ready for another version of base.

Definition 5.9 (s-base). An s-base (for spanning base) of a (supertropical) vector space $V$ is a minimal tropical spanning set $S$, in the sense that no proper subset of $S$ tropically spans $V$.

As we shall see in Examples 5.22 below, a vector space with a finite d-base could still fail to have an s-base. Even when an s-base exists, it could be considerably larger than any d-base.

Example 5.10. Elements of a vector space $V$ may be tropically dependent on a subspace $W$ but not tropically spanned by $W$, as indicated in Example 5.7(i).

Example 5.11. Let $V$ be the subspace of $D(\mathbb{R})^{(2)}$ spanned by

$$
S=\left\{(1,1),\left(1^{v}, 1\right),\left(1,1^{v}\right)\right\}
$$

in logarithmic notation, equipped with the standard ghost module. Each of these vectors alone comprises a d-base of $V$, whereas $S$ is an s-base of $V$.

An s-base $S$ need not be finite. On the other hand, obviously any finite tropical spanning set contains an s-base, so any finitely spanned vector space has an sbase. In order to coordinate the definitions of s-base and d-base, we introduce the following definition.

Definition 5.12. A $d, s$-base is an s-base which is also independent, that is, also is a d-base. A supertropical vector space $V$ is finite-dimensional if it has a finite d,s-base. 
Proposition 5.13. The cardinality of the $d, s$-base $S$, if finite, is precisely $\operatorname{rk}(V)$.

Proof. $|S| \geq \operatorname{rk}(V)$ by Proposition 5.8. But we get equality, since by definition $S$ is itself a d-base.

Example 5.14. Suppose $S$ is a tropically independent subset of $V$. Then $S$ is a d,s-base of the subspace of $V$ tropically spanned by $S$. These are the subspaces of greatest interest to us.

Example 5.15. There are four possible sorts of nonzero subspaces of $F^{(2)}$ tropically spanned by a set $S$ of tangible elements over a supertropical semifield $F$, writing $\left\{\varepsilon_{1}=\left(\mathbb{1}_{F}, \mathbb{O}_{F}\right), \varepsilon_{2}=\left(\mathbb{O}_{F}, \mathbb{1}_{F}\right)\right\}$ for the standard base:

(i) The plane $F^{(2)}$ itself.

(ii) A half-plane of tangible rank 2, having tangible s-base containing $\varepsilon_{1}$ or $\varepsilon_{2}$, as well as one tangible element $\alpha_{1} \varepsilon_{1}+\alpha_{2} \varepsilon_{2}$ for $\alpha_{1}, \alpha_{2} \in \mathscr{T}$.

(iii) A planar strip of tangible rank 2, having tangible s-base

$$
\left\{\alpha_{1} \varepsilon_{1}+\alpha_{2} \varepsilon_{2}, \beta_{1} \varepsilon_{1}+\beta_{2} \varepsilon_{2}\right\},
$$

where $\alpha_{1}, \alpha_{2}, \beta_{1}, \beta_{2} \in \mathscr{T}$.

(iv) A subspace of tangible rank 1, each pair of whose elements is tropically dependent. The tangible vectors are all multiples of a single vector.

One also has examples of nontangibly generated subspaces of $F^{(2)}$, such as $W=\left\{\left(\alpha, \alpha^{\nu}\right): \alpha \in F\right\}$.

Here are some examples of strange behavior of s-bases, when we reverse the direction in tropical spanning in a supertropical vector space $V$. For subsets $S, S^{\prime} \subset V$, we say that $S^{\prime}$ ghost surpasses $S$ if for each $v \in S^{\prime}$, there is some $v^{\prime} \in S^{\prime}$ such that $v^{\prime} \models_{\text {gs }} v$.

Examples 5.16. (i) Let $V$ be the vector space tropically spanned by the vectors $v_{1}, v_{2}, v_{3}$ of Example 5.7(i), and let $W$ be the subspace tropically spanned by the vectors $v_{1}$ and $v_{2}$. Note that

$$
v_{1}+v_{2}=\left(1^{v}, 1,1\right) \models_{\mathrm{gs}}(0,1,1)=v_{3} .
$$

We claim more generally that $W$ ghost surpasses $V$. Indeed, by symmetry we may consider $v=(\alpha, \beta, \gamma) \in V$ with $\alpha \leq_{v} \beta \leq_{v} \gamma$. By the definition of $V$, we must have either $\beta \cong_{\nu} \gamma$ or $\gamma=\gamma^{\nu}$. In the former case, $\beta\left(v_{1}+v_{2}\right) \models_{\mathrm{gs}} v$, and in the latter case, $v \models_{\mathrm{gs}} \beta v_{1}+\gamma v_{3}$.

(ii) Here is an example of a supertropical space $V$ with an s-base $\left\{v_{1}, v_{2}, v_{3}, v\right\}$ in which the subspace $W$ tropically spanned by $v_{1}, v_{2}$, and $v_{3}$ ghost surpasses $\{v\}$, and 
the subspace $W^{\prime}$ tropically spanned by $v_{1}$ and $v_{2}$ ghost surpasses $\left\{v_{3}\right\}$, but $W^{\prime}$ does not ghostsurpass $\{v\}$. Let

$$
\begin{aligned}
& v_{1}=(2,2,0,2,2,0), \quad v_{2}=(2,0,2,2,0,2), \\
& v_{3}=(0,2,2,0,2,2), \quad v=(2,2,2,1,1,1) .
\end{aligned}
$$

Then $v_{1}+v_{2}+v_{3} \models_{\mathrm{gs}} v$ and $v_{1}+v_{2} \models_{\mathrm{gs}} v_{3}$, but we claim that $W^{\prime}$ does not ghost surpass $\{v\}$. Suppose $\alpha_{1} v_{1}+\alpha_{2} v_{2} \models_{\mathrm{gs}} v$. If $\alpha_{1}=\alpha_{2}$, then from the second component, $\alpha_{1}=0$, so from the fifth component $\alpha_{2}=2$ : a contradiction.

Thus $\alpha_{1} \neq \alpha_{2}$. By symmetry, we may assume that $\alpha_{1}>\alpha_{2}$. Then from the first component, $\alpha_{1}=-2$, and now we have a contradiction from the second component.

(iii) We now exhibit a space $V$ in which the s-base $\mathscr{B}$ has 6 vectors and which is ghost surpassed by a set of 4 vectors; but if one removes any single vector from $\mathscr{B}$ it fails to ghost surpass $V$. Take

$$
\begin{aligned}
& v_{1}=(4,4,0,2,2,0), \quad v_{2}=(4,0,4,2,0,2), \quad v_{3}=(0,4,4,0,2,2), \\
& w_{1}=(2,2,0,2,2,0), \quad w_{2}=(2,0,2,2,0,2), \quad w_{3}=(0,2,2,0,2,2) \text {. }
\end{aligned}
$$

The vector $(4,4,4,3,3,3)$ is not ghost surpassed by any 5 of these vectors.

Critical elements versus s-bases. Since s-bases are involved in the actual generation of the space, they are more in tune with the classical theory of convexity, and can be studied combinatorially. Here is another way to view the s-base, which is inspired by the literature on convex spaces. We say that two elements $v, w$ in a supertropical vector space $V$ over a supertropical semifield $F$ are projectively equivalent, written $v \sim w$, if and only if $v=\alpha w$ for some tangible element $\alpha \in F$. Accordingly, we define the equivalence class of $v$ as

$$
[v]_{\sim}:=\{w \in V \mid w \sim v\} .
$$

Definition 5.17. A vector $v \notin \mathscr{H}_{0}$ in a supertropical vector space $V$ is critical if we cannot write $v \models_{\mathrm{gs}} v_{1}+v_{2}$ for $v_{1}, v_{2} \in V \backslash[v]_{\sim}$. Taking one representative for each class $[v] \sim$, a critical set of $V$ is defined as a set of representatives of all the critical elements of $V$.

Critical elements correspond to "extreme points" over the max-plus algebra in [Gaubert and Katz 2007], in which it is shown that every point in $F^{(n)}$ is a linear combination of at most $n+1$ extreme points.

Example 5.18. Consider the space $V$ spanned by the five critical vectors

$$
\begin{array}{ll}
(0,-\infty, 0,-\infty, 0,-\infty), & (-\infty, 0,-\infty, 0,-\infty, 0), \\
(0,-\infty,-\infty, 0,-\infty,-\infty), & (-\infty, 0,-\infty,-\infty, 0,-\infty), \\
(-\infty,-\infty, 0,-\infty,-\infty, 0) . &
\end{array}
$$


Then $(0,0,0,0,0,0)$ is the sum of the first two vectors as well as the last three.

There is a basic connection between criticality and almost tangible.

Lemma 5.19. Suppose $v \models_{\mathrm{gs}} \alpha v+w$ for $\alpha \in \mathcal{T}, v, w \in V$. Then $\alpha \leq_{v}$ e. Moreover:

(1) If $\alpha<_{v} e$, then $v \models_{\mathrm{gs}} w$.

(2) Suppose $\alpha \in \mathscr{T}_{e}$, that is, $\alpha \cong_{\nu}$ e. If $w \in \mathscr{H}_{\mathbb{D}}$, then $v=\alpha v$. For any $w \in V$,

$$
v=\alpha^{2} v+e w^{\prime}=\alpha^{2} v
$$

where $w^{\prime} \models_{\mathrm{gs}} w$.

Proof. Write $v=\alpha v+w^{\prime}$, where $w^{\prime} \models_{\mathrm{gs}} w$.

If $\alpha>_{\nu} e$, then

$$
v=\alpha v+w^{\prime}=\left(\alpha+\mathbb{1}_{F}\right) v+w^{\prime}=v+\alpha v+w^{\prime}=v+v=e v \in \mathscr{H}_{\mathbb{D}} .
$$

But then $v=v+\alpha v+w^{\prime}$, implying by Proposition 2.14 that $v=v+\alpha v=\alpha v$, and thus $\alpha \cong_{\nu} e$.

(1) If $\alpha<_{\nu} e$, then $\mathbb{1}_{F}=\alpha+\mathbb{1}_{F}$, implying

$$
v=\left(\alpha+\mathbb{1}_{F}\right) v=\alpha v+v=\alpha v+\alpha v+w^{\prime}=e \alpha v+w^{\prime} \models_{\mathrm{gs}} w,
$$

proving (1).

(2) Thus, we assume that $\alpha \in \mathscr{T}_{e}$. If $w=e w$, then

$v=\alpha v+w^{\prime}=\alpha\left(\alpha v+w^{\prime}\right)+w^{\prime}=\alpha^{2} v+\left(\alpha+\mathbb{1}_{F}\right) w^{\prime}=\alpha^{2} v+e w^{\prime}=\alpha\left(\alpha v+e w^{\prime}\right)=\alpha v$.

For any $w$, if $\alpha \in \mathscr{T}_{e}$, then

$$
v=\alpha v+w^{\prime}=\alpha\left(\alpha v+w^{\prime}\right)+w^{\prime}=\alpha^{2} v+\left(\alpha+\mathbb{1}_{F}\right) w^{\prime}=\alpha^{2} v+e w^{\prime} .
$$

Hence, $v=\alpha^{2} v$ by the preceding argument, replacing $w$ by $e w^{\prime}$.

Proposition 5.20. Any critical element $v \in V$ is almost tangible.

Proof. Otherwise $v=w+w^{\prime}$ for suitable $w \in V, w^{\prime} \in \mathscr{H}_{\mathbb{D}}$, for which $w \notin \mathscr{T}_{e} v$, but by criticality, $w=\alpha v$ for $\alpha \in \mathcal{T}$. Then, by Lemma 5.19, $\alpha \leq_{v} e$, and furthermore $\alpha \in \mathscr{T}_{e}$, since otherwise $v \models_{\mathrm{gs}} e w^{\prime}$, contrary to $v \notin \mathscr{H}_{\mathbb{D}}$. But now, by Lemma 5.19, $v=\alpha v=w$, a contradiction.

Lemma 5.21. An almost tangible element $v \in V$ is critical if and only if it is not tropically spanned by $V \backslash[v]_{\sim}$, that is, $v \not \#_{\mathrm{gs}} \sum \alpha_{i} w_{i}$ for any $\alpha_{i} \in \mathscr{T}, w_{i} \in V \backslash[v]_{\sim}$.

Proof. The "if" part is immediate by definition. To prove the converse, suppose on the contrary that $v \models_{\mathrm{gs}} \sum_{i=1}^{t} \alpha_{i} w_{i}$; by definition of criticality, $t>1$. Then taking $v_{1}=\alpha_{1} w_{1}$ and $v_{2}=\sum_{i=2}^{t} \alpha_{i} w_{i}$, we must have $v_{2} \in[v]_{\sim}$, and conclude by induction on $t$. 
Clearly a critical set of a vector space $V$ is projectively unique, but could be empty.

Examples 5.22. (i) The standard base $\left\{\varepsilon_{1}, \ldots, \varepsilon_{n}\right\}$ of $F^{(n)}$ is also its critical set.

(ii) The critical set of the subspace $W=F^{(2)} \backslash\left(\left[\varepsilon_{1}\right]_{\sim} \cup\left[\varepsilon_{2}\right]_{\sim}\right)$ is empty.

(iii) $W=F^{(2)} \backslash\left[\varepsilon_{1}\right] \sim$ has the critical set $\left[\varepsilon_{2}\right] \sim$, but has no s-base.

Despite the last two examples, some positive information is available.

Lemma 5.23. Any tropical spanning set $S$ contains a tropical critical set of $V$.

Proof. Suppose $v \in V$ is critical. By hypothesis on $S, v$ is tropically spanned by $S$, but by Lemma 5.21, it must be an element of $S$ (up to projective equivalence).

Theorem 5.24. Suppose $V$ has an s-base $S$. Then $S$ is a critical set of $V$.

Proof. In view of Lemma 5.23, it remains to show that each element of $S$ is critical. Suppose $v \in S$ is not critical. Then $v=v_{1}+v_{2}$ where $v_{1}, v_{2} \notin \mathscr{T} v$. Thus, when we write

$$
v_{1}=\sum_{i} \alpha_{1, i} s_{1, i}+w_{1} \quad \text { and } \quad v_{2}=\sum_{i} \alpha_{2, i} s_{2, i}+w_{2}
$$

for $\alpha_{1, i}, \alpha_{2, i} \in \mathscr{T}, s_{1, i}, s_{2, i} \in S$, and $w_{1}, w_{2} \in \mathscr{H}_{\mathbb{0}}$, we must have $v$ appearing in one of the sums (for otherwise $v=v_{1}+v_{2}$ is tropically spanned by the other elements of $S$, contrary to hypothesis).

Thus, we may assume $s_{1,1}=v$, and we have

$$
v_{1} \models_{\mathrm{gs}} \alpha_{1} v+\sum_{i \neq 1} \alpha_{1, i} s_{1, i},
$$

and similarly $v_{2} \models_{\mathrm{gs}} \alpha_{2} v+\sum_{i \neq 1} \alpha_{2, i} s_{2, i}$. (Formally, we permit $\alpha_{2}=\mathbb{O}_{F}$.) We also write $v_{j}=\alpha_{j}+x_{j}$, where $x_{j} \models_{\mathrm{gs}} \sum_{i \neq 1} \alpha_{j, i} s_{j, i}$.

Now

$$
v=v_{1}+v_{2}=\beta v+x,
$$

where $\beta=\alpha_{1,1}+\alpha_{2,1}$ and $x=x_{1}+x_{2}$. But then $\beta \leq_{v} e$, by Lemma 5.19, which also says that if $\beta<_{v} e$, then $v \models_{\mathrm{gs}} x$, contrary to $S$ being an s-base. Thus, we may conclude that $\beta \cong_{\nu} e$. By symmetry, we assume that $\alpha_{1} \cong_{\nu} e$. If $\alpha_{2}<_{\nu} e$, then $v_{2}=_{\mathrm{gs}} x_{2}$, and

$$
\begin{aligned}
v & =v_{1}+v_{2}=\alpha_{1} v+x_{1}+v_{2}=\alpha_{1}\left(\alpha_{1} v+x_{1}+v_{2}\right)+x_{1}+v_{2} \\
& =\alpha_{1}^{2} v+\left(\alpha_{1}+\mathbb{1}_{F}\right)\left(x_{1}+v_{2}\right)=\alpha_{1}^{2} v+e\left(x_{1}+v_{2}\right),
\end{aligned}
$$

and thus

$$
\begin{aligned}
v_{1} & =\alpha_{1}^{2} v+e\left(x_{1}+v_{2}\right)=\alpha_{1}\left(\alpha_{1}^{2} v+e\left(x_{1}+v_{2}\right)\right)+x_{1}+v_{2} \\
& =\alpha_{1}^{3} v+e\left(x_{1}+v_{2}\right)=\alpha_{1}\left(\alpha_{1}^{2} v+e\left(x_{1}+v_{2}\right)\right)=\alpha_{1} v .
\end{aligned}
$$


Thus, we are done for $\alpha_{2}<_{v} e$, and may assume that $\alpha_{2} \in \mathscr{T}_{e}$. Then

$$
v=\left(\alpha_{1}+\alpha_{2}\right) v+x_{1}+x_{2}=e v+x,
$$

implying $v=e x \in \mathscr{H}$ and thus $\alpha_{j} v=\alpha_{j} e v=e v$ for $j=1$, 2. Hence

$$
v=v_{1}+v_{2}=\alpha_{1} v+x_{1}+\alpha_{2} v+x_{2}=\left(\alpha_{1}+\alpha_{2}\right) v+x=e v+x,
$$

and thus $v=e v+e x$ by Lemma 5.19, implying

$$
\begin{aligned}
v_{1} & =e v+x_{1}=e v+e x+x_{1}=e v+e x_{1}+e x_{2}+x_{1} \\
& =e v+e x_{1}+e x_{2}=e v+e x=v .
\end{aligned}
$$

Thus, we have the following striking result:

Corollary 5.25. The s-base (if it exists) of a supertropical vector space is unique up to multiplication by tangible elements of $F$, and is comprised of almost tangible elements.

Example 5.26. The only s-bases of the supertropical vector space $V=F^{(n)}$ are its classical bases $S=\left\{\alpha_{1} \varepsilon_{1}, \ldots, \alpha_{n} \varepsilon_{n}\right\}$, where $\alpha_{1}, \ldots, \alpha_{n} \in \mathcal{T}$.

One also has the following tie between critical sets and s-bases.

Proposition 5.27. Any critical set $C$ of a supertropical vector space $V$ is an s-base of the subspace $W$ tropically spanned by $C$.

Proof. By hypothesis, $C$ tropically spans $W$, so we need only check minimality. But for any $v \in C$, by definition, $C \backslash\{v\}$ does not tropically span $v$.

Definition 5.28 (thick subspace). A subspace $W$ of a supertropical vector space $V$ is thick if $\operatorname{rk}(W)=\operatorname{rk}(V)$.

For example, any subspace of $F^{(n)}$ containing $n$ tropically independent vectors is thick.

Remark 5.29. By definition, any thick subspace of a thick subspace of $V$ is thick in $V$.

Remark 5.30. Any thick subspace $W$ of a supertropical vector space $V$ contains a d-base of $V$. Indeed, by definition, for $n=\operatorname{rk}(V), W$ contains a set of $n$ tropically independent elements, which must be a maximal tropically independent set in $V$, by definition of rank. Thus, $V$ is tropically dependent on any thick subspace.

Example 5.31. There exists an infinite chain of thick subspaces $W_{1} \subset W_{2} \subset \cdots$ of $V=D(\mathbb{R})^{(2)}$, where $W_{k}$ is the strip tropically spanned by $\{(k, 0),(0, k)\}, k \in \mathbb{N}^{+}$. Thus, $\{(k, 0),(0, k)\}$ is not an s-base of $D(\mathbb{R})^{(2)}$. (One could expand this to an uncountable chain by taking $k \in \mathbb{R}^{+}$.)

Note that projectively a thick subspace need not be either convex in the classical sense or of pure dimension [Izhakian et al. 2011]. 
5A. Change of base matrices. We write $P_{\pi}$ for the permutation matrix whose entry in the $(i, \pi(i))$ position is $\mathbb{1}_{F}$ (for each $1 \leq i \leq n$ ) and $\mathbb{O}_{F}$ elsewhere. Likewise, we write $\operatorname{diag}\left\{a_{1}, \ldots, a_{n}\right\}$ for the diagonal matrix whose entry in the $(i, i)$ position is $a_{i}$ and $\mathbb{O}_{F}$ elsewhere, and denote it as $D$. We call the product $P_{\pi} D$ of a permutation matrix and a tangible (nonsingular) diagonal matrix, with each diagonal entry $\neq \mathbb{O}_{F}$, a generalized permutation matrix, and denote it as $\widetilde{P}_{\pi ; D}$.

Recall from [Izhakian and Rowen 2011a, Proposition 3.9] that over a supertropical semifield, a matrix is invertible if and only if it is a generalized permutation matrix $\widetilde{P}_{\pi ; D}$ with $D$ nonsingular. In particular, the set of all generalized permutation matrices form a group whose unit element is $I$.

Definition 5.32. Given s-bases $\mathscr{B}=\left\{v_{1}, \ldots, v_{n}\right\}$ and $\mathscr{B}^{\prime}=\left\{v_{1}^{\prime}, \ldots, v_{n}^{\prime}\right\}$ of $V \subseteq F^{(n)}$, whose respective row matrices are denoted $A$ and $A^{\prime}$, a change of base matrix is a matrix $P$ such that

$$
A^{\prime}=P A \text {. }
$$

Proposition 5.33. The generalized permutation matrices are the only change of base matrices of s-bases (and thus classical bases).

Proof. Immediate by Corollary 5.25.

Remark 5.34. It follows from Proposition 5.33, applied to the standard base, that the matrix $A$ is the matrix of a classical base if and only if $A$ is a generalized permutation matrix.

\section{Supertropical bilinear forms}

The classical way to study orthogonality in vector spaces is by means of bilinear forms. In this section, we introduce the supertropical analog, providing some of the basic properties. Although the tropical literature deals with orthogonality in terms of the inner product, as described in [Akian et al. 2006, §25.6], the supertropical theory leads to a more axiomatic approach.

The notion of supertropical bilinear form follows the classical algebraic theory, although, as is to be expected, there are a few surprises, mostly because of the characteristic 2 nature of the theory [Izhakian et al. 2013]. In this section, we assume that $V$ is a vector space over a supertropical semifield $F$.

\section{Supertropical bilinear forms.}

Definition 6.1. A (supertropical) bilinear form on supertropical vector spaces $V$ and $V^{\prime}$ is a function $B: V \times V^{\prime} \rightarrow F$ satisfying

$$
\begin{gathered}
B\left(v_{1}+v_{2}, w_{1}+w_{2}\right) \models_{\mathrm{gs}} B\left(v_{1}, w_{1}\right)+B\left(v_{1}, w_{2}\right)+B\left(v_{2}, w_{1}\right)+B\left(v_{2}, w_{2}\right), \\
B(\alpha v, w)=\alpha B(v, w)=B(v, \alpha w),
\end{gathered}
$$


for all $\alpha \in F, v_{i} \in V$, and $w_{j} \in V^{\prime}$. We say that a bilinear form $B$ is strict if

$$
\begin{aligned}
& B\left(\alpha_{1} v_{1}+\alpha_{2} v_{2}, \beta_{1} w_{1}+\beta_{2} w_{2}\right) \\
& \quad=\alpha_{1} \beta_{1} B\left(v_{1}, w_{1}\right)+\alpha_{1} \beta_{2} B\left(v_{1}, w_{2}\right)+\alpha_{2} \beta_{1} B\left(v_{2}, w_{1}\right)+\alpha_{2} \beta_{2} B\left(v_{2}, w_{2}\right),
\end{aligned}
$$

for all $v_{i} \in V$ and $w_{i} \in V^{\prime}$.

When $V^{\prime}=V$, we say that $B$ is a (supertropical) bilinear form on the vector space $V$.

We usually write $\langle v, w\rangle$ in place of $B(v, w)$. We do not assume that $\langle v, w\rangle=$ $\langle w, v\rangle$. For the remainder of this section, we take $V^{\prime}=V \subseteq F^{(n)}$, and consider a (supertropical) bilinear form $B$ on $V$.

Perhaps surprisingly, one can lift many of the classical theorems about bilinear forms to the supertropical setting, without requiring strictness.

Definition 6.2. The Gram matrix of vectors $v_{1}, \ldots, v_{k} \in V=F^{(n)}$ is defined as the $k \times k$ matrix

$$
\widetilde{G}\left(v_{1}, \ldots, v_{k}\right)=\left(\begin{array}{cccc}
\left\langle v_{1}, v_{1}\right\rangle & \left\langle v_{1}, v_{2}\right\rangle & \cdots & \left\langle v_{1}, v_{k}\right\rangle \\
\left\langle v_{2}, v_{1}\right\rangle & \left\langle v_{2}, v_{2}\right\rangle & \cdots & \left\langle v_{2}, v_{k}\right\rangle \\
\vdots & \vdots & \ddots & \vdots \\
\left\langle v_{k}, v_{1}\right\rangle & \left\langle v_{k}, v_{2}\right\rangle & \cdots & \left\langle v_{k}, v_{k}\right\rangle
\end{array}\right) .
$$

The set $\left\{v_{1}, \ldots, v_{k}\right\}$ is nonsingular (with respect to $B$ ) if and only if its Gram matrix is nonsingular (see Section 3). The Gram matrix of $V$ is the Gram matrix of an s-base of $V$.

Example 6.3. The quasi-identity

$$
\widetilde{G}\left(v_{1}, v_{2}\right)=\left(\begin{array}{cc}
0 & 1^{v} \\
-\infty & 0
\end{array}\right)
$$

(in logarithmic notation) is the Gram matrix of a bilinear form. Note that

$$
\left\langle v_{1}, v_{2}\right\rangle=1^{v}>0^{v}=\left\langle v_{1}, v_{1}\right\rangle+\left\langle v_{2}, v_{2}\right\rangle .
$$

In particular, we have the matrix $\widetilde{G}=\widetilde{G}\left(b_{1} \ldots, b_{k}\right)$, which can be written as $\left(g_{i, j}\right)$ where $g_{i, j}=\left\langle b_{i}, b_{j}\right\rangle$; we describe the bilinear form via the matrix equation

$$
\langle v, w\rangle \models_{\mathrm{gs}} v^{\mathrm{t}} \widetilde{G} w .
$$

Of course, the matrix $\widetilde{G}$ depends on the choice of tangible s-base $\mathscr{B}$ of $V$, but this is unique up to multiplication by scalars and permutation, so $\widetilde{G}$ is unique up to $\widetilde{P}_{\pi ; D} \widetilde{G} \widetilde{P}_{\pi ; D}^{t}$, where $\widetilde{P}_{\pi ; D}$ is a generalized permutation matrix. In particular, whether or not $\widetilde{G}$ is nonsingular does not depend on the choice of s-base. 
Ghost orthogonality. Bilinear forms play a key role in geometry since they permit us to define orthogonality of supertropical vectors. However, as we shall see, orthogonality is rather delicate in this setup. We work with a fixed bilinear form $B=\langle$,$\rangle on a supertropical vector space V$.

Definition 6.4. For vectors $v, w$ in $V$, we write $v \Perp w$ when $\langle v, w\rangle \in \mathscr{G}_{\mathbb{D}}$, that is, $\left\langle w_{1}, w_{2}\right\rangle \models_{\mathrm{gs}} \mathbb{O}_{F}$ (see Remark 2.5), and say that $v$ and $w$ are left ghost orthogonal, or lg-orthogonal for short. For subspaces $W_{1}, W_{2}$ of $V$, we say that $W_{1}$ is lg-orthogonal to $W_{2}$ if every $w_{1} \in W_{1}$ is lg-orthogonal to every $w_{2} \in W_{2}$.

The left orthogonal ghost complement $S^{\Perp}$ of $S$ is defined as

$$
S^{\Perp}:=\left\{v \in V:\langle v, S\rangle \in \mathscr{G}_{\mathbb{O}}\right\} .
$$

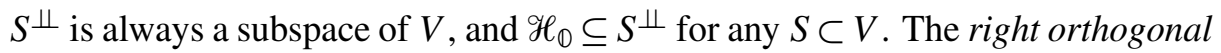
ghost complement is defined analogously. Of course, when $\langle v, w\rangle \in \mathscr{H}_{\mathbb{D}}$ implies $\langle w, v\rangle \in \mathscr{H}_{\mathbb{D}}$, we may omit the words "left" and "right" and talk of g-orthogonality, which then is a symmetric relation.

Definition 6.5. A subspace $W$ of $V$ is called nondegenerate (with respect to $B$ ) if $W^{\Perp} \cap W \subseteq \mathscr{H}_{0}$. The bilinear form $B$ is nondegenerate if the space $V$ is nondegenerate.

Lemma 6.6. Suppose $\left\{w_{1}, \ldots, w_{m}\right\}$ tropically spans a subspace $W$ of $V$, and $v \in V$. If $\sum_{i=1}^{m} \beta_{i}\left\langle v, w_{i}\right\rangle \in \mathscr{G}_{\mathbb{0}}$ for all $\beta_{i} \in \mathscr{T}$, then $v \in W^{\Perp}$.

Proof. $\left\langle v, \sum_{i} \beta_{i} w_{i}\right\rangle \models_{\mathrm{gs}} \sum_{i}\left\langle v, \beta_{i} w_{i}\right\rangle=\sum_{i} \beta_{i}\left\langle v, w_{i}\right\rangle \in \mathscr{G}_{\mathbb{0}}$ for all $\beta_{i} \in \mathcal{T}$. Thus, $v \in W^{\Perp}$.

Theorem 6.7. Assume that the vectors $w_{1}, \ldots, w_{k} \in V$ span a nondegenerate subspace $W$ of $V$. If $\left|\widetilde{G}\left(w_{1} \ldots, w_{k}\right)\right| \in \mathscr{G}_{\mathbb{0}}$, then $w_{1} \ldots, w_{k}$ are tropically dependent.

Proof. Write $\widetilde{G}=\widetilde{G}\left(v_{1}, \ldots, v_{k}\right)$. By [Izhakian and Rowen 2011a, Theorem 6.6], $|\widetilde{G}| \in \mathscr{G}_{\mathbb{0}}$ if and only if the rows of $\widetilde{G}$ are tropically dependent. By the lemma, if $|\widetilde{G}| \in \mathscr{G}_{\mathbb{O}}$, then some linear combination of the $w_{i}$ is in $W^{\Perp}$. When $W$ is nondegenerate, this latter assertion is the same as saying that the $w_{i}$ are tropically dependent.

Corollary 6.8. If the bilinear form $B$ is nondegenerate on a vector space $V$, then the Gram matrix (with respect to any given supertropical d,s-base of $V$ ) is nonsingular.

Remark 6.9. In case the bilinear form $B$ is strict, we can strengthen Lemma 6.6 to obtain, for $v \in V$,

$$
v \in W^{\Perp} \Longleftrightarrow \sum_{i=1}^{m} \beta_{i}\left\langle v, w_{i}\right\rangle \in \mathscr{G}_{\mathbb{0}} \text { for all } \beta_{i} \in \mathscr{T} .
$$

(Indeed, if $v \in W^{\Perp}$, then $\sum_{i} \beta_{i}\left\langle v, w_{i}\right\rangle=\left\langle v, \sum_{i} \beta_{i} w_{i}\right\rangle \in \mathscr{G}_{\mathbb{0}}$ for all $i$.) 
In this case, we can also strengthen Corollary 6.8 to read:

Corollary 6.10. A strict bilinear form B is nondegenerate on a supertropical vector space $V$ if and only if the Gram matrix (with respect to any given supertropical $d, s$-base of $V$ ) is nonsingular.

\section{Symmetry of g-orthogonality.}

Definition 6.11. The bilinear form $B$ is supertropically alternate if $\langle v, v\rangle \in \mathscr{G}_{\mathbb{0}}$ for all $v \in V . B$ is symmetric if $\langle v, w\rangle=\langle w, v\rangle$ for all $v, w \in V . B$ is supertropically symmetric if $\langle v, w\rangle \Upsilon_{\mathrm{gd}}\langle w, v\rangle$ for all $v, w \in V$, that is, $\langle v, w\rangle+\langle w, v\rangle \in \mathscr{G}_{\mathbb{D}}$.

In this subsection, we prove the supertropical version of a classical theorem of Artin, that any bilinear form in which g-orthogonality is symmetric must be a supertropically symmetric bilinear form. (In characteristic 2, any alternate form is symmetric, so we would expect our supertropical forms in the conclusion of the theorem to be symmetric in some sense.)

Definition 6.12. The (supertropical) bilinear form $B$ is orthogonal-symmetric if it satisfies the following property for all $v_{i}, w \in V$ :

$$
\sum_{i}\left\langle v_{i}, w\right\rangle \in \mathscr{G}_{\mathbb{0}} \Longleftrightarrow \sum_{i}\left\langle w, v_{i}\right\rangle \in \mathscr{G}_{\mathbb{0}}
$$

for any finite sum taken over $v_{i} \in V$.

$B$ is supertropically orthogonal-symmetric if $B$ is orthogonal-symmetric and satisfies the additional property that $\langle v, w\rangle \cong_{\nu}\langle w, v\rangle$ for all $v, w \in V$ satisfying $\langle v, w\rangle \in \mathscr{T}$.

Remark 6.13. If every $\langle v, v\rangle=\mathbb{O}_{F}$, for all $v \in V$, then the (supertropical) bilinear form $B$ is trivial. (Indeed, $\mathbb{O}_{F}=\langle v+w, v+w\rangle=\langle v, w\rangle+\langle w, v\rangle$ for all $v, w \in V$, implying $\langle v, w\rangle=\langle w, v\rangle=\mathbb{O}_{F}$.)

Thus we need to modify our notion of isotropic.

Definition 6.14. A vector $v \in V$ is g-isotropic if $\langle v, v\rangle \in \mathscr{G}_{\mathbb{D}}$.

Lemma 6.15. When the bilinear form $B$ is strict, Condition (6-4) reduces to the condition

$$
\langle v, w\rangle \in \mathscr{G}_{\mathbb{0}} \Longleftrightarrow\langle w, v\rangle \in \mathscr{G}_{\mathbb{0}} .
$$

Proof. Taking $v=\sum_{i} v_{i}$, we have

$$
\sum_{i}\left\langle v_{i}, w\right\rangle=\langle v, w\rangle, \quad\langle w, v\rangle=\sum_{i}\left\langle w, v_{i}\right\rangle
$$

The symmetry condition extends to sums. 
Lemma 6.16. If $B$ is supertropically symmetric, then

$$
\sum_{i}\left\langle v_{i}, w\right\rangle \in \mathscr{T} \Longleftrightarrow \sum_{i}\left\langle w, v_{i}\right\rangle \in \mathscr{T} .
$$

In this case, $\sum_{i}\left\langle v_{i}, w\right\rangle=\sum_{i}\left\langle w, v_{i}\right\rangle$.

Proof. We may assume that $\sum_{i}\left\langle v_{i}, w\right\rangle \in \mathcal{T}$ and $\sum_{i}\left\langle w, v_{i}\right\rangle \in \mathcal{T}$, since there is nothing to check if one (and thus the other) is ghost. Take $i_{1}$ such that $\left\langle v_{i_{1}}, w\right\rangle$ is the dominant summand of $\sum_{i}\left\langle v_{i}, w\right\rangle$, and thus is tangible. Likewise, take $i_{2}$ such that $\left\langle w, v_{i_{2}}\right\rangle$ is the dominant summand of $\sum_{i}\left\langle w, v_{i}\right\rangle$, and thus is tangible. By hypothesis, $\left\langle v_{i_{1}}, w\right\rangle=\left\langle w, v_{i_{1}}\right\rangle$ and $\left\langle w, v_{i_{2}}\right\rangle=\left\langle v_{i_{2}}, w\right\rangle$, since these are tangible. Since these dominate their respective sums, we get $\sum_{i}\left\langle v_{i}, w\right\rangle=\sum_{i}\left\langle w, v_{i}\right\rangle \in \mathscr{T}$.

We aim to prove that a g-orthogonal-symmetric (supertropical) bilinear form is supertropically symmetric.

Remark 6.17. The condition $\langle v, w\rangle+\langle w, v\rangle \in \mathscr{G}_{\mathbb{O}}$ means that $v$ is g-orthogonal to $w$ with respect to the new (symmetric) bilinear form given by $\langle v, w\rangle^{\prime}:=\langle v, w\rangle+\langle w, v\rangle$.

Lemma 6.18. Suppose that $B$ is a g-orthogonal-symmetric bilinear form and $v, w \in V$. Then either $\langle v, w\rangle+\langle w, v\rangle \in \mathscr{G}_{\mathbb{O}}$, or $\langle v, v\rangle=\langle w, w\rangle=\mathbb{O}_{F}$.

Proof. One may assume that $\langle v, w\rangle \in \mathscr{T}$; hence $\langle w, v\rangle \in \mathscr{T}$. If $\langle v, w\rangle \cong_{v}\langle w, v\rangle$, then $\langle v, w\rangle+\langle w, v\rangle \in \mathscr{G}_{\mathbb{0}}$, so we may assume by symmetry that $\langle v, w\rangle>_{v}\langle w, v\rangle$.

First assume that $w$ is non-g-isotropic. Then $\gamma\langle v, w\rangle+\langle w, w\rangle$ is ghost for $\gamma=\langle w, w\rangle /\langle v, w\rangle$ and tangible for any other tangible $\gamma$ in $F$. At the same time, $\gamma\langle w, v\rangle+\langle w, w\rangle$ is ghost for $\gamma=\langle w, w\rangle /\langle w, v\rangle$, contradicting g-orthogonalsymmetry unless $\langle v, w\rangle \cong_{v}\langle w, v\rangle$. This implies $\langle v, w\rangle+\langle w, v\rangle \in \mathscr{G}_{\mathbb{D}}$.

Next assume that $w$ is g-isotropic but $\langle w, w\rangle=\alpha^{v} \neq \mathbb{O}_{F}$ for $\alpha \in \mathscr{T}$. Then for tangible $\gamma>_{v}\langle w, w\rangle /\langle v, w\rangle$, we see that $\langle\gamma v, w\rangle+\langle w, w\rangle$ is tangible, so $\langle w, \gamma v\rangle+\langle w, w\rangle$ must also be tangible, which is false if $\gamma<_{v}\langle w, w\rangle /\langle w, v\rangle$. This yields a contradiction if $\langle w, v\rangle<_{v}\langle v, w\rangle$, and similarly we have a contradiction if $\langle w, v\rangle>_{\nu}\langle v, w\rangle$; hence $\langle w, v\rangle \cong_{\nu}\langle v, w\rangle$, implying $\langle v, w\rangle+\langle w, v\rangle \in \mathscr{G}_{\mathbb{D}}$.

Thus, we may assume that $\langle w, w\rangle=\mathbb{O}_{F}$. Likewise, $\langle v, v\rangle=\mathbb{O}_{F}$, since otherwise we would conclude by interchanging $v$ and $w$.

We conclude with our supertropical version of Artin's theorem.

Theorem 6.19. Every g-orthogonal-symmetric bilinear form $B$ on a supertropical vector space $V$ is supertropically symmetric.

Proof. We are done by Lemma 6.18 unless there are vectors $v, w \in V$ for which $\langle v, v\rangle=\langle w, w\rangle=\mathbb{O}_{F}$ and $\langle v, w\rangle+\langle w, v\rangle \in \mathscr{T}$.

In this case, $\alpha:=\langle v, w\rangle \in \mathscr{T}, \beta:=\langle w, v\rangle \in \mathscr{T}$, and $\alpha+\beta \in \mathscr{T}$. Observe that, if $v^{\prime} \in V$ such that $\left\langle v^{\prime}, w\right\rangle \cong_{\nu} \alpha$, then $\left\langle w, v^{\prime}\right\rangle \cong_{\nu} \beta$. Indeed, $\langle v, w\rangle+\left\langle v^{\prime}, w\right\rangle=\alpha^{v}$, implying $\langle w, v\rangle+\left\langle w, v^{\prime}\right\rangle \in \mathscr{G}_{\mathbb{0}}$. But $\left\langle w, v^{\prime}\right\rangle \in \mathscr{T}$, so we conclude that $\left\langle w, v^{\prime}\right\rangle \cong_{\nu} \beta$. 
Now let vector $v^{\prime}$ be any vector of $V$. Then

$$
\left\langle v+v^{\prime}, w\right\rangle \models_{\mathrm{gs}}\langle v, w\rangle+\left\langle v^{\prime}, w\right\rangle \neq \mathbb{0}_{F} .
$$

Thus, $\left\langle v+v^{\prime}, w\right\rangle \cong_{v} \gamma$, for some $\gamma \in \mathscr{T}$. Let $v^{\prime \prime}:=(\alpha / \gamma)\left(v+v^{\prime}\right)$. Then

$$
\left\langle v^{\prime \prime}, w\right\rangle=\frac{\alpha}{\gamma}\left\langle v+v^{\prime}, w\right\rangle \cong_{\nu} \alpha,
$$

and thus $\left\langle w, v^{\prime \prime}\right\rangle \cong_{v} \beta$, as just observed. Hence, $\left\langle v^{\prime \prime}, w\right\rangle+\left\langle w, v^{\prime \prime}\right\rangle \notin \mathscr{G}$. Now Lemma 6.18 yields $\left\langle v^{\prime \prime}, v^{\prime \prime}\right\rangle=\mathbb{O}_{F}$. From

$$
\mathbb{O}_{F}=\left\langle\gamma v^{\prime \prime}, \gamma v^{\prime \prime}\right\rangle \models_{\mathrm{gs}}\langle v, v\rangle+\left\langle v, v^{\prime}\right\rangle+\left\langle v^{\prime}, v\right\rangle+\left\langle v^{\prime}, v^{\prime}\right\rangle,
$$

we conclude that $\left\langle v^{\prime}, v^{\prime}\right\rangle=\mathbb{O}_{F}$ for all $v^{\prime} \in V$; i.e., $B$ is trivial, by Remark 6.13, which is absurd since $\alpha=\langle v, w\rangle \neq \mathbb{O}_{F}$. Thus, $B$ must be supertropically symmetric.

Corollary 6.20. If the bilinear form B is strict and g-orthogonality is a symmetric relation, then $B$ is supertropically symmetric.

Proof. $B$ is orthogonal-symmetric, by Lemma 6.15. The theorem then can be applied.

\section{References}

[Akian et al. 1990] M. Akian, G. Cohen, S. Gaubert, R. Nikoukhah, and J. P. Quadrat, "Linear systems in (Max-+) algebra", pp. 151-156 in Proceedings of the 29th Conference on Decision and Control (Honolulu, 1990), Institute of Electrical and Electronics Engineers, New York, 1990.

[Akian et al. 2006] M. Akian, R. Bapat, and S. Gaubert, "Max-plus algebra”, Chapter 25 in Handbook of linear algebra, edited by L. Hogben et al., Discrete Mathematics and its Applications 39, Chapman and Hall/CRC, Boca Raton, FL, 2006.

[Akian et al. 2009] M. Akian, S. Gaubert, and A. Guterman, "Linear independence over tropical semirings and beyond", pp. 1-38 in Tropical and idempotent mathematics, edited by G. L. Litvinov and S. N. Sergeev, Contemp. Math. 495, Amer. Math. Soc., Providence, RI, 2009. MR 2011e:16085 Zbl 1182.15002

[Feichtner and Sturmfels 2005] E. M. Feichtner and B. Sturmfels, "Matroid polytopes, nested sets and Bergman fans”, Port. Math. (N.S.) 62:4 (2005), 437-468. MR 2006j:05036 Zbl 1092.52006 arXiv math/0411260v1

[Gaubert and Katz 2007] S. Gaubert and R. D. Katz, "The Minkowski theorem for max-plus convex sets”, Linear Algebra Appl. 421:2-3 (2007), 356-369. MR 2007k:52001 Zbl 1110.52002

[Izhakian 2009] Z. Izhakian, "Tropical arithmetic and matrix algebra", Comm. Algebra 37:4 (2009), 1445-1468. MR 2010d:16059 Zbl 1165.15017 arXiv math.AG/0505458

[Izhakian and Rowen 2009] Z. Izhakian and L. Rowen, "The tropical rank of a tropical matrix", Comm. Algebra 37:11 (2009), 3912-3927. MR 2010k:15003 Zbl 1184.15003

[Izhakian and Rowen 2010] Z. Izhakian and L. Rowen, "Supertropical algebra", Adv. Math. 225:4 (2010), 2222-2286. MR 2012a:14137 Zbl 05796760 arXiv 0806.1171

[Izhakian and Rowen 2011a] Z. Izhakian and L. Rowen, "Supertropical matrix algebra", Israel J. Math. 182 (2011), 383-424. MR 2012e:16108 Zbl 1215.15018 
[Izhakian and Rowen 2011b] Z. Izhakian and L. Rowen, "Supertropical matrix algebra II: Solving tropical equations", Israel J. Math. 186 (2011), 69-96. MR 2852317 Zbl 06108394 arXiv 0902.2159

[Izhakian and Rowen 2011c] Z. Izhakian and L. Rowen, "Supertropical matrix algebra, III: Powers of matrices and their supertropical eigenvalues", J. Algebra 341 (2011), 125-149. MR 2012e:15054 Zbl 06005428 arXiv 1008.0023

[Izhakian et al. 2011] Z. Izhakian, M. Johnson, and M. Kambites, "Pure dimension and projectivity of tropical convex sets", preprint, 2011. arXiv 1106.4525

[Izhakian et al. 2013] Z. Izhakian, M. Knebusch, and L. Rowen, "Supertropical quadratic forms, I", preprint, 2013. arXiv 1309.5729

[Maclagan and Sturmfels 2009] D. Maclagan and B. Sturmfels, "Introduction to tropical geometry", 2009, http://homepages.warwick.ac.uk/staff/D.Maclagan/papers/TropicalBook.html.

Received August 4, 2010. Revised November 20, 2012.

ZUR IZHAKIAN

DEPARTMENT OF MATHEMATICS

BAR-ILAN UNIVERSITY

RAMAT-GAN 52900

ISRAEL

zzur@math.biu.ac.il

MANFRED KNEBUSCH

DEPARTMENT OF MATHEMATICS

UNIVERSITY OF REGENSBURG

REGENSBURG

GERMANY

manfred.knebusch@mathematik.uni-regensburg.de

LOUIS ROWEN

DEPARTMENT OF MATHEMATICS

BAR-ILAN UNIVERSITY

RAMAT-GAN 52900

ISRAEL

rowen@macs.biu.ac.il 


\title{
PACIFIC JOURNAL OF MATHEMATICS
}

\author{
msp.org/pjm
}

Founded in 1951 by E. F. Beckenbach (1906-1982) and F. Wolf (1904-1989)

\section{EDITORS}

V. S. Varadarajan (Managing Editor)

Department of Mathematics

University of California

Los Angeles, CA 90095-1555

pacific@math.ucla.edu

Paul Balmer

Department of Mathematics

University of California

Los Angeles, CA 90095-1555

balmer@math.ucla.edu

Daryl Cooper

Department of Mathematics

University of California

Santa Barbara, CA 93106-3080 cooper@math.ucsb.edu

Jiang-Hua $\mathrm{Lu}$

Department of Mathematics

Pokfulam Rd., Hong Kong jhlu@maths.hku.hk
The University of Hong Kong

Don Blasius

Department of Mathematics University of California

Los Angeles, CA 90095-1555

blasius@math.ucla.edu

Robert Finn

Department of Mathematics Stanford University

Stanford, CA 94305-2125

finn@math.stanford.edu

Sorin Popa

Department of Mathematics

University of California

Los Angeles, CA 90095-1555 popa@math.ucla.edu

Paul Yang

Department of Mathematics Princeton University

Princeton NJ 08544-1000

yang@math.princeton.edu

\section{PRODUCTION}

Silvio Levy, Scientific Editor, production@msp.org

\section{SUPPORTING INSTITUTIONS}

ACADEMIA SINICA, TAIPEI

CALIFORNIA INST. OF TECHNOLOGY

INST. DE MATEMÁTICA PURA E APLICADA

KEIO UNIVERSITY

MATH. SCIENCES RESEARCH INSTITUTE

NEW MEXICO STATE UNIV.

OREGON STATE UNIV.

\author{
STANFORD UNIVERSITY \\ UNIV. OF BRITISH COLUMBIA \\ UNIV. OF CALIFORNIA, BERKELEY \\ UNIV. OF CALIFORNIA, DAVIS \\ UNIV. OF CALIFORNIA, LOS ANGELES \\ UNIV. OF CALIFORNIA, RIVERSIDE \\ UNIV. OF CALIFORNIA, SAN DIEGO \\ UNIV. OF CALIF., SANTA BARBARA
}

\author{
Vyjayanthi Chari \\ Department of Mathematics \\ University of California \\ Riverside, CA 92521-0135 \\ chari@math.ucr.edu \\ Kefeng Liu \\ Department of Mathematics \\ University of California \\ Los Angeles, CA 90095-1555 \\ liu@math.ucla.edu \\ Jie Qing \\ Department of Mathematics \\ University of California \\ Santa Cruz, CA 95064 \\ qing@cats.ucsc.edu
}

These supporting institutions contribute to the cost of publication of this Journal, but they are not owners or publishers and have no responsibility for its contents or policies.

See inside back cover or msp.org/pjm for submission instructions.

The subscription price for 2013 is US \$400/year for the electronic version, and \$485/year for print and electronic.

Subscriptions, requests for back issues and changes of subscribers address should be sent to Pacific Journal of Mathematics, P.O. Box 4163, Berkeley, CA 94704-0163, U.S.A. The Pacific Journal of Mathematics is indexed by Mathematical Reviews, Zentralblatt MATH, PASCAL CNRS Index, Referativnyi Zhurnal, Current Mathematical Publications and the Science Citation Index.

The Pacific Journal of Mathematics (ISSN 0030-8730) at the University of California, c/o Department of Mathematics, 798 Evans Hall \#3840, Berkeley, CA 94720-3840, is published twelve times a year. Periodical rate postage paid at Berkeley, CA 94704, and additional mailing offices. POSTMASTER: send address changes to Pacific Journal of Mathematics, P.O. Box 4163, Berkeley, CA 94704-0163.

PJM peer review and production are managed by EditFLOW ${ }^{\circledR}$ from Mathematical Sciences Publishers.

\section{PUBLISHED BY}

mathematical sciences publishers

nonprofit scientific publishing

http://msp.org/

(C) 2013 Mathematical Sciences Publishers 


\section{PACIFIC JOURNAL OF MATHEMATICS}

Volume $266 \quad$ No. $1 \quad$ November 2013

Multiplicity of solutions to the Yamabe problem on collapsing $\quad 1$ Riemannian submersions

Renato G. Bettiol and Paolo Piccione

Rank gradient of small covers

DARLAN GIRÃO

Nonrationality of nodal quartic threefolds

KYUSIK HONG

Supertropical linear algebra

ZUR IZHAKIAN, MANFRED KNEBUSCH and LOUIS ROWEN

Isometry groups among topological groups

PIOTR NIEMIEC

Singularities and Liouville theorems for some special conformal

Hessian equations

QIANZHONG OU

Attaching handles to Delaunay nodoids

FRANK PACARD and HAROLD ROSENBERG

Some new canonical forms for polynomials

BRUCE REZNICK

Applications of the deformation formula of holomorphic one-forms 